\title{
Supporting Information: Discovering new lipidomic features using cell type specific fluorophore expression to provide spatial and biological specificity in a multimodal workflow with MALDI Imaging Mass Spectrometry
}

Marissa A. Jones $\uparrow+$, Sung Hoon Cho§, Nathan Heath Patterson $\neq \|$, Raf Van de Plas $\ddagger \| \perp$, Jeffrey

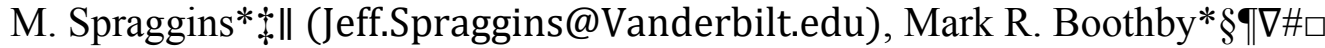

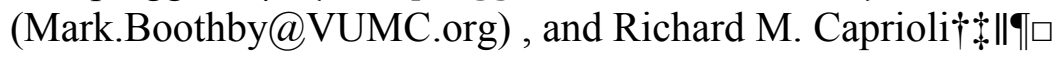

$†$ Department of Chemistry, Vanderbilt University, 7330 Stevenson Center, Station B 351822, Nashville, Tennessee 37235

\$ Mass Spectrometry Research Center and Department of Biochemistry, Vanderbilt University Medical Center, 465 21st Avenue South, MRB III Suite 9160, Nashville, Tennessee 37232.

$\S$ Department of Pathology, Microbiology, and Immunology, $116121^{\text {st }}$ Avenue South, MCN AA-4214B, MCN A5301, Vanderbilt University Medical Center, Nashville, TN 37232.

॥Department of Biochemistry, Vanderbilt University, Nashville, TN 37232.

$\perp$ Delft Center for Systems and Control (DCSC), Delft University of Technology, 2628 CD Delft, The Netherlands qDepartment of Medicine, Vanderbilt University, Nashville, TN

$\nabla$ Department of Cancer Biology, Vanderbilt University, Nashville, TN

\#Vanderbilt-Ingram Cancer Center, Vanderbilt University, Nashville, TN

$\square$ Pharmacology, Vanderbilt University, Nashville, TN

\section{Table of Contents}

Extended Methods: S1-S9

Supplemental Figure Legends: S10-S12

Supplemental Figures: S13-S46 


\section{SI Appendix}

\section{Materials}

MALDI matrix 1,5-diaminonapthalene (DAN) was purchased from Sigma-Aldrich Chemical Co. (St. Louis, MO, USA). Sheep red blood cells (SRBC), ammonium formate, carboxymethyl cellulose sodium salt, isopropyl alcohol, mass spectrometry grade water, chloroform, and acetonitrile were purchased from Fisher Scientific (Pittsburg, PA, USA); streptavidin-Alexa647 antibody (Ab) and chemically conjugated monoclonal Ab (GL7-FITC, $\alpha$ IgD-PE and $\alpha$ CD35biotin) were purchased from BD Biosciences (San Jose, CA). C57BL/6-J mice and breeding stock transgenic for a bacterial artificial chromosome that integrates a translational fusion of GFP with AID into the Aicda locus (AID-GFP mice; stock\# 018421) were obtained from Jackson Laboratory and bred with C57BL/6-J. All mice were housed in ventilated microisolators under Specified-Pathogen-Free conditions in a Vanderbilt mouse facility and used in accordance with protocols approved by the Institutional Animal Care \& Use Committee.

\section{Tissue Preparation}

Mice were used at age six to seven weeks and were euthanized eight days postimmunization. AID-GFP and C57BL/6-J mice were immunized with SRBC $\left(2 \times 10^{8}\right.$ intraperitoneally) to compare with non-immunized controls (C57BL/6-J mice, $n=3$ ). Spleens were extracted, snap-frozen on dry ice in tissue molds, and stored at $-80{ }^{\circ} \mathrm{C}$ prior to sectioning. Samples were post-embedded in degassed $2.6 \%(\mathrm{w} / \mathrm{v})$ carboxymethyl cellulose over dry ice. Tissues were sectioned at $12 \mu \mathrm{m}$ using a cryostat (Leica $3050 \mathrm{~S}$ ) at $-17{ }^{\circ} \mathrm{C}$ and thaw-mounted onto ITO (Delta Technologies) or Super Frost Gold Plus microscope slides (Fisher Scientific). Three serial sections with one section of each sample type were placed on triads of slides with 
the central slide (ITO) destined for IMS and the peripheral slides (Super Frost Gold Plus) used for $\mathrm{H} \& \mathrm{E}$ and IF. After sectioning, slides were placed in a desiccator for 15 minutes prior to any secondary imaging modality. $\mathrm{F}_{\mathrm{em}}$ was performed on all sections and slides. All fluorescence microscopy data were collected using a Nikon 90i (Nikon, Melville, NY, USA), and then used for alignment and identification of GCs via detection of $\mathrm{F}_{\mathrm{em}}$ of transgenic fluorophore. FITC, TRITC, and DAPI filters were used, all with 150 msec exposures at 10x magnification and autofocusing in the GFP channel. After $\mathrm{F}_{\mathrm{em}}$ scanning, ammonium formate washing was performed to remove excess salts and enhance IMS signal. Samples on ITO slides for IMS were washed four times (each for $15 \mathrm{sec}$, without agitation) in $50 \mathrm{mM}$ ammonium formate $\left(\mathrm{pH}\right.$ of 6.4 at $4{ }^{\circ} \mathrm{C}$ ) and then placed in a desiccator $(15 \mathrm{~min})$ to ensure drying before matrix application ${ }^{42}$.

Concurrently, IF was performed on one serial section and H\&E staining was performed on a second serial section. For IF, frozen tissue sections were air dried (1 min) and rehydrated (two times, each for $1 \mathrm{~min}$ ) in ice cold $\left(4^{\circ} \mathrm{C}\right)$ phosphate-buffered saline (PBS). Slides were then fixed with fresh $1 \%$ paraformaldyde for 10 minutes and washed (three times, each for $2 \mathrm{~min}$ ) with PBS and then treated with M.O.M. blocking buffer $\left(1\right.$ hour at $\left.20^{\circ} \mathrm{C}\right)($ Vector Lab; Burlingame, CA). Sections were then incubated ( 3 hours at $4^{\circ} \mathrm{C}$ ) with $25-30 \mu \mathrm{L}$ of primary antibody [ $\alpha$-GL7-FITC (1:50), $\alpha$-IgD-PE (1:200), and $\alpha$-CD35-biotin (1:200)], washed 3 times with PBS, followed by incubation with streptavidin-Alexa 647 (1:200) in blocking buffer for 1-2 hours at $4{ }^{\circ} \mathrm{C}$ and again washing $3 \mathrm{x}$ with PBS and mounting with Prolong Diamond anti-fade reagent (Thermo Fisher, Waltham, MA). Immunohistochemistry scans were collected using an Aperio Versa 200 using DAPI, FITC, Cy3, and Cy5 at 10x with exposure time optimized for each sample. 
For H\&E, sections were fixed in $95 \%$ EtOH followed by $70 \% \mathrm{EtOH}$ (30 sec each) and rinsed in Milli-Q water until clear. Sections were first stained in hematoxylin (2.5 min), rinsed in water, dipped in $0.5 \%$ ammonium hydroxide and rinsed in water, and then dipped 20 times in $70 \% \mathrm{EtOH}$ followed by $95 \%$ ethanol 20 times. After staining with eosin for 1 minute, slides were further prepared through 20 dips in 95\% ethanol and a second fresh wash in 95\% ethanol for 10 seconds. Slides were then moved to a $100 \%$ ethanol solution for 20 dips twice and then placed in xylenes for 2 minutes prior to cover slipping.

For the IMS modality, matrix was applied to ammonium formate-washed samples with a robotic sprayer (TM Sprayer, HTX, Chapel Hill, NC, USA) operated at a speed of $1200 \mathrm{~mm} / \mathrm{min}$ with a track spacing of $1.5 \mathrm{~mm}$, using nitrogen as a flow gas at 10 PSI. Recrystallized 1,5-DAN was dissolved in a 9:1 (v/v) solution of acetonitrile/deionized water at a concentration of 10 $\mathrm{mg} / \mathrm{mL}$ and sonicated $15 \mathrm{~min}$. The sprayer nozzle was held at $85^{\circ} \mathrm{C}$ to deposit matrix in eight “criss-cross" passes at a flow rate of $0.15 \mathrm{~mL} / \mathrm{min}$ with a pushing solvent of $90 / 10(\mathrm{v} / \mathrm{v})$ acetonitrile/ $\mathrm{H}_{2} \mathrm{O}$ for an average density of $6.667 \mu \mathrm{g} / \mathrm{mm}^{2}$.

\section{IMS}

Negative ion mode IMS data were acquired with a 9.4T Bruker FT-ICR SolariX mass spectrometer (Bruker Daltonics, Billerica, MA, USA) equipped with a $2 \mathrm{kHz} \mathrm{Nd}$ : YAG (wavelength: $355 \mathrm{~nm}$ ) laser and an Infinity ion cyclotron resonance (ICR) cell. Laser power was optimized for each sample by adjusting in the range of $18-20 \%$. Data were collected in negative ion mode from $\mathrm{m} / \mathrm{z} 200-2,000$ with a raster step of $30 \mu \mathrm{m}$ and a data size of $1 \mathrm{MB}$ per spectrum with a free induction decay (FID) length of 0.8389 s. Each pixel consisted of 500 laser shots with the minimum laser energy focus setting $(\sim 10 \mu \mathrm{m})$ at a frequency of $2000 \mathrm{~Hz}$, and a smart walk of 
$25 \mu \mathrm{m}$ was enabled to increase sensitivity. The mass-selecting quadrupole was set to $\mathrm{m} / \mathrm{z} 350$ and TOF of $0.8 \mathrm{~ms}$. The resolving power of all imaging experiments was $\sim 80,000$ at $\mathrm{m} / z 766.54$. External calibration was performed prior to analysis and internal lock-mass calibration was performed using $m / z 885.5494$ ([PI(18:0_20:4)-H]-) during image acquisition. For image fusion analysis, a higher spatial resolution image was generated using the same 9.4T FT-ICR with similar settings except that the raster step was $15 \mu \mathrm{m}$ without smart walk enabled, and 750 laser shots per pixel were generated at a laser power of $13 \%$. Datasets are available at: https://figshare.com/s/ab2f73880453100e0c2c.

\section{Image Registration}

Fundamentals of image registration - the transformation of a group of images onto a single coordinate plane in as close of a match as possible - have been described in a supplement to published work ${ }^{16}$. For registration, source images were transformed to match the corresponding target images - in this work, the IMS coordinate system with the source image being the other modalities connected through $\mathrm{F}_{\mathrm{em}}$ (Fig. 1e, Supplemental Table 4). This transformation involved some deformation of images determined through an iterative function towards improved alignment. Due to the need to integrate multi-modal data, we used the affine registration model with a mean distance error at $\sim 1 \mu \mathrm{m}$ for same-section registration and $\sim 10 \mu \mathrm{m}$ for registration of two adjacent sections, with the minimum image resolution as the limiting factor in each case $^{16}$.

\section{Registration Experimental Considerations}

All modalities were aligned to IMS data on a per pixel basis, by linking of the theoretical pixel location in each image to the laser ablation marks made by the IMS laser in a raster across 
the tissue. Both single-section multi-image, and multi-section multi-image workflows were used in registration ${ }^{16}$. Images were exported as high-resolution jpeg, tiff, mha, or png files. All images were registered to a pixel map, or theoretical grid, generated from a coordinate extraction from flex imaging, using a padding of 10 to add area to the images around the edges (Supplemental Fig. 6, Supplemental Table 4). We used external fiducial marks (rectangular regions external to the tissue) to reduce inaccuracy that could be introduced in determination of the exact center of the ablation mark for alignment to the theoretical pixel map of tissue at high spatial resolution (Supplemental Table 4). These fiducial benchmarks were acquired at half the spatial resolution and were used to enable high accuracy alignment with the center of the theoretical pixel map obtained from the spot list map. Laser ablation sites post-acquisition as well as external fiducial marks were recorded in the FITC channel. Masks in FIJI were used to increase accuracy of registration ${ }^{43}$, and image figures were created using FIJI, using its pre-programmed macros.

\section{IMS Data Analyses}

To quantify overlap (congruence) in two adjacent sections across entire data sets, samples from immunized AID-GFP transgenic mice were annotated for GC localization in FIJI using regions of interest and imported into $\mathrm{R}^{44,45}$. A custom script was used to determine the Sorenson dice coefficient between each serial section, and the coefficients determined for each section pair were then averaged (Supplemental Fig. 1 and Supplemental Table 1). IMS data were initially preprocessed by importation into SCiLS and root mean square normalized. These normalized data were then converted to. imzml format and imported in the $\mathrm{R}$ environment via Cardinal package. Images were generated in $\mathrm{R}$ and overlaid with all other modalities. Data were further analyzed through manual interpretation, spatially shrunken centroid segmentation with the 
Cardinal package, and via image fusion to enable correlation of IMS m/z channels to RGB channels in a partial least squares model.

For manual interpretation, each ion image was screened visually for its relationship to the GC images in side by side comparisons, and only those ions that appeared to localize specifically were selected. For spatially shrunken centroid data analysis, data were exported into R by importing data into SCiLS and enacting extraction of peak lists via the file tab "export SCiLS report table" (Supplemental Fig. 7). This was accomplished by importing the data into R in the correct format to be read into Cardinal using a home-built R script (Supplemental File 1). Data were then segmented until $\mathrm{k}$ means clustering produced a GC-like segment. Each ion image was analyzed separately, and the top $\mathrm{m} / \mathrm{z}$ ions for each segment with their weights were exported into Excel for further analysis. The ions with a $\mathrm{T}$ statistic above 15 were selected due to previous manual interpretation results showing that both $\mathrm{m} / \mathrm{z} 776.5596$ and m/z 752.5591 were GCspecific ions. Ions were only tested statistically to determine whether they were present in all three biological replicates in this refined list (Supplemental Table 2).

For image fusion analysis with IMS data sets, the mass spectrometry data were treated as a data cube in which the $\mathrm{x}$ and $\mathrm{y}$ coordinates are pixel dimensions and the $\mathrm{z}$ coordinate is $\mathrm{m} / \mathrm{z}$. Analogously, the microscopy data map pixel dimensions are $\mathrm{x}$ and $\mathrm{y}$, but the $\mathrm{z}$ coordinate was the color channels. Image fusion algorithms were then used in two phases - creation of a crossmodality model and then use of the model to perform partial least squares regression correlation to compare image pairs of IMS and $\mathrm{F}_{\mathrm{em}}$ data. In the present work, relationships were mined to identify ions of interest that related to a specific color channel, in this case green to correlate IMS with the AID-GFP fluorophore. Further information on this algorithm can be found in previous work $^{18,46}$. IMS data collection for image fusion processing was performed with $15 \mu \mathrm{m}$ raster 
steps in negative ion mode using sectioned (12 $\mu \mathrm{m}$ thickness) spleen of immunized AID-GFP transgenic mice. The mass range was truncated to $\mathrm{m} / \mathrm{z} 500$ to 900 , and the top 119 most abundant ions were selected for analysis. The white box correlation model was broken open to identify key ions directly and inversely correlating with the FITC channel in $\mathrm{F}_{\mathrm{em}}$ microscopy images acquired prior to the IMS. A full implementation of the image fusion framework is provided as a command-line utility that can be downloaded at http://fusion.vueinnovations.com/. Additional information on preformatting data for image fusion can be found at https://github.com/NHPatterson/aimsMSRC (Supplemental Table 4).

\section{QuPath: region specific annotation and weighted average intensity extraction}

To enable statistical analyses, variance was determined for ions of interest discovered through manual interpretation, segmentation, and image fusion. Quantitative Pathology and Bio image analysis (QuPath) software ${ }^{23}$ was used to annotate and extract ion intensities from the MALDI imaging datasets. Samples were manually annotated in QuPath, adjusting contrast as needed for GCs to identify the light and dark zones (Supplemental Fig. 8). Data were then extracted using an R script in which pixels with a 50\% overlap were included in the region of interest and a weighted average (weighted by IMS pixel - annotation ROI overlap) was taken for each such region (Supplemental file 2). To test the null hypothesis - no genuine difference in distribution of the $\mathrm{m} / \mathrm{z}$ species - these averages and their variances were then compared using ratio $\mathrm{T}$ tests (Table 1). GC ions were selected based on their significance and then to determine whether these ions were specific to GCs. Once GC co-localizing ions - defined based on results of such statistical testing in comparing GC to all other regions interest - were determined, these $\mathrm{m} / \mathrm{z}$ features were further probed using serial sections to distinguish the two GC subregions. For 
these latter analyses, we used only GC for which both light and dark zones were clearly identified. These pairs were selected using an R script in which Euclidean distance was used to establish the nearest neighbor within 5 contiguous pixels (approximately $67 \mathrm{um}^{2}$ ) (Supplemental file 2). These weighted averages were then further tested for significance with ratio $T$ tests.

\section{Identification of Lipid Species}

To specify the lipids that corresponded to the GC-enriched ions, provisional identifications were made by comparing accurate masses of the precursor ions and fragment ions to theoretical $\mathrm{m} / \mathrm{z}$ values in LIPID MAPS (Nature Lipidomics Gateway, http://www.lipidmaps.org/ ). For further validation by LC-MS/MS, data were acquired with a $\mathrm{Q}$ Exactive HF mass spectrometer linked in negative ion mode from m/z $375-1650$ in PRM mode with an isolation window of 2 Da for each ion of interest using eluates from a Vanquish UHPLC (Thermo Scientific, Waltham, MA, USA). MS/MS resolving power was 15,000 at $\mathrm{m} / \mathrm{z} 200$, while full scan resolving power was at $\mathrm{m} / \mathrm{z} 200$ was 30,000 . Chromatography was performed on a Waters BEH C8 column (150mm x 2, $1 \mathrm{~mm}$ x 1, $7 \mu \mathrm{m}$ particle size, Waters, Milford, MA, USA) using solvent $\mathrm{A}[10 \mathrm{mM}$ ammonium acetate in water/acetonitrile $(1: 1 \mathrm{v} / \mathrm{v})]$ and solvent $\mathrm{B}[10$ $\mathrm{mM}$ ammonium acetate in isopropyl alcohol/acetonitrile $(1: 1 \mathrm{v} / \mathrm{v})]$, eluting with a gradient as follows: Solvent B was held at $20 \%$ B for 1 min followed by a gradient over 8 min to $100 \%$ solvent B. Solvent B was held at 100\% B for 2 min and then decreased to $20 \%$ B in 0.5 minutes followed by an isocratic solution at 2\% B for 4.5 minutes. Resultant LC-MS/MS data were analyzed manually using Xcalibur Qual Browser (Thermo Xcalibur 2.2 SP1.48, Thermo Fisher Scientific Inc., Waltham, MA, USA) and Lipid Maps MS tools (Nature Lipidomics Gateway, http://www.lipidmaps.org/tools/ms/). 
Complementary analyses were then performed using MS/MS-based imaging experiments. Tandem MS/MS data from IMS were acquired using a 15T Bruker FT-ICR solariX mass spectrometer (Bruker Daltonics, Billerica, MA, USA). The instrument is equipped with a Smartbeam II 2kHz Nd: YAG (wavelength: $355 \mathrm{~nm}$ ) laser and a Paracell ICR cell. The raster step of the laser was set to $60-120 \mu \mathrm{m}$. Data were collected in negative ion mode from $m / z 250-2,000$ with a data size of $1 \mathrm{MB}$ per spectrum. Each pixel consisted of 1,000 laser shots with the minimum setting of the laser $(\sim 10 \mu \mathrm{m})$ at a frequency of $2000 \mathrm{~Hz}$ and power of $40-80 \%$. Ions of interest were isolated with a 2-6 Da mass window and fragmented using collision induced dissociation (CID) with a collision energy of $17-27 \mathrm{~V}$ in the accumulation multipole prior to transfer to the ICR cell for mass analysis (Example method Supplemental File 3). 


\section{SUPPLEMENTAL FIGURE LEGENDS}

Supplemental Figure 1. Serial section accuracy: a visual representation. The $F_{\mathrm{em}}$ images from two registered serial sections of mouse spleen after SRBC immunization depicted in a) were used to annotate AID-GFP GC. b) Masks of these annotations were generated, and c) the overlap between these annotations is visually shown in white with an Dice-Sorenson coefficient (DSC) of 0.85 . To the right a color legend is shown to indicate the section mask, overlap, and tissue outline. A scale bar can be found in white indicating $1000 \mu \mathrm{m}$. See supplemental Table 1 for more information.

Supplemental Figure 2. High accuracy registration of richer multimodal data. a) Shown are representative registered images highlighting the types of detection and nature of splenic samples. Sample types included: immunized and non-immunized (Imm, Non-Imm) nontransgenic mice (WT) as well as AID-GFP transgenic mice (AID-GFP Imm). Rectangular areas of spleen are shown with each section, from left to right, conventional hematoxylin and eosin (H\&E), Fluorescence Emission ( $\left.F_{\mathrm{em}}\right)$, Immunofluorescence (IF), IMS with three ions overlaid for context showcasing $\mathrm{m} / \mathrm{z} 776.5596, \mathrm{~m} / \mathrm{z} 791.5410$, and $\mathrm{m} / \mathrm{z} 810.5269$, a single ion image highlighting ion intensity differences within the sub-region for the ion $m / z 776.5596$ (IMS ${ }_{776}$ ), a similar overlay of ions $m / z 752.5591,791.5410$, and 810.5269, and a similar single ion image showing $m / z 752.5591$ (IMS 752$)$. Fem was taken on the same section imaged by IMS. IF and H\&E images were then taken from serial sections. IF was used to identify micro-anatomic portions of lymphoid follicles, and included both indirect and direct staining of GL7, IgD, and CD35 as described in the Methods. On the far right intensity scales for each ion image showing the 
percentage of total ion count can be found as well as a color legend for immunofluorescence images. Size is indicated by a scale bar embedded in each H\&E image shows $1000 \mu \mathrm{m}$. b) Magnified regions showing a single germinal center for each sample type are shown with the same sample order and modalities. The region from which these were magnified is designated in region $1 \mathrm{a}$ by a white box.

Supplemental Figure 3. A step-wise general workflow for annotation and data extraction of germinal center light and dark zones pairs annotated in QuPath. a) IF samples were loaded into QuPath and the light zone, indicated in blue, and the DZ, indicated in yellow, are shown. b) The mouse spleen was annotated manually. c) A zoom in of a single annotation with both DZ and LZ sub regions is shown. d) GC pairs were detected by Euclidian distance. e) Data was extracted from GC pairs with LZs and DZs larger than 5 contiguous pixels and statistical analyses were performed.

\section{Supplemental Figure 4. Application of image fusion algorithm substantially increased the} yield of GC correlated lipids. a) Both modalities, $F_{\mathrm{em}}$ and IMS are separated into color variables for $\mathrm{F}_{\mathrm{em}}$ and $\mathrm{m} / \mathrm{z}$ variables for IMS data as shown in call-out graphs and mass spectra. b) Data-driven image fusion ${ }^{18}$ using a multivariate linear regression model was performed. c) A cross modality model between IMS data and the red, green, and blue channels of $\mathrm{F}_{\mathrm{em}}$ data. c) This model enables the correlation of IMS ions to the color channels of $F_{\mathrm{em}}$. By mining the data for ions that show a high correlation to the green channel in $\mathrm{F}_{\mathrm{em}}$, ions correlated to $\mathrm{GC}$ identified by GFP were extracted $\mathbf{d})$. 
Supplemental Figure 5. Ion images of the top GC ions were detected through a data-driven image fusion relating IMS to $\mathbf{F}_{\mathrm{em}}$. a) Magnified regions of $\mathrm{F}_{\mathrm{em}} / \mathrm{AF}$ and $\mathbf{b}$ ) IF are shown to identify germinal centers. A scale bar indicating $1000 \mu \mathrm{m}$ is shown in the $\mathrm{F}_{\mathrm{em}} / \mathrm{AF}$ image. Shown in c) through r) magnified ion images of GC related species listed in Table 1.

\section{Supplemental Figure 6. Screenshots illustrating how to extract the spot list and make a} pixel map. a) Within FlexImaging, a Bruker IMS data analysis tool, to extract the spot list, go to file -> export $->$ SpotList. b) That spot list is then converted into a pixel map within the MSRC Autofluoresence Registration Toolbox. A screenshot showing the parameters used in this experiment are shown.

Supplemental Figure 7. An example of a SCiLS report table for exporting peak values for Cardinal in RStudio. To export the .imzml equivalent of Bruker data to Rstudio, a report table converted into a .csv is used. This includes centroid $m / z$ values and a \pm Da window for each peak.

Supplemental Figure 8. An example of the QuPath annotation platform. In QuPath, annotations of germinal centers and their light and dark zones were made for exporting weighted mean values of ions for these regions.

\section{Supplemental Figure 9-27. Imaging MS/MS Validation of GC correlating and} anticorrelating ions. Selected ion images of selected fragments of each lipid shown in table 1 and Fig 3. Fragmentation data for PE(O-18:0_20:4) and PE(O-18:0_22:6) can be found in Fig.4. Lipids were identified using the lipid maps MS/MS spectrum prediction and MS/MS data search. 


\section{Supplemental Figure 1}

Serial Section 1 Serial Section 2

Pre IMS $\mathrm{F}_{\mathrm{er}}$ Pre IMS F

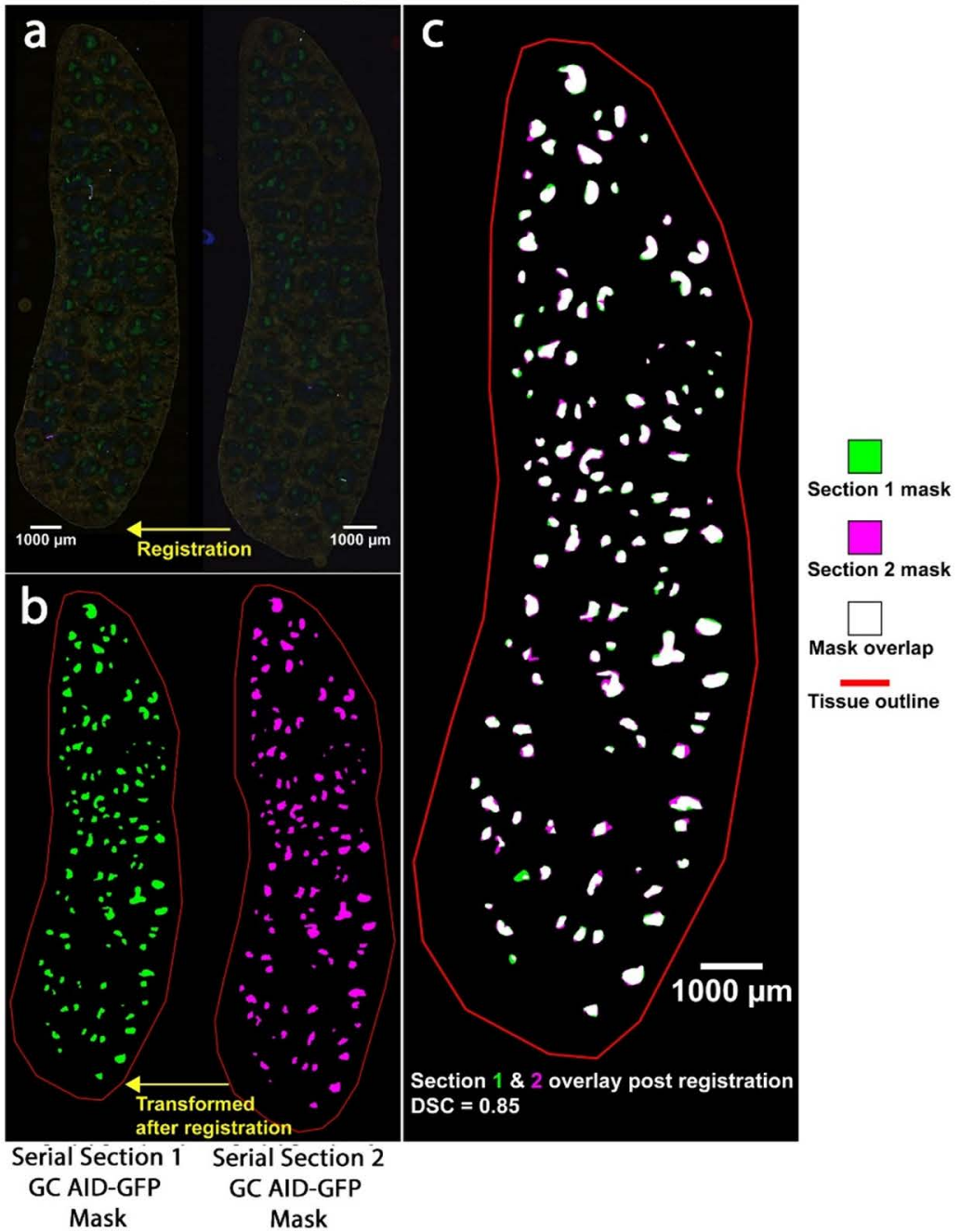




\section{Supplemental Figure 3}

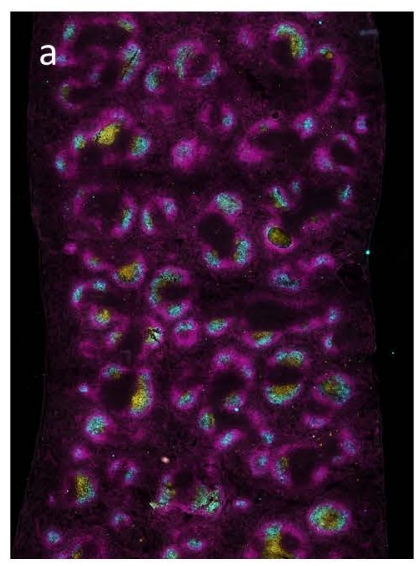

Mouse Spleen IF $\square$ Light Zone $\square$ Dark Zone

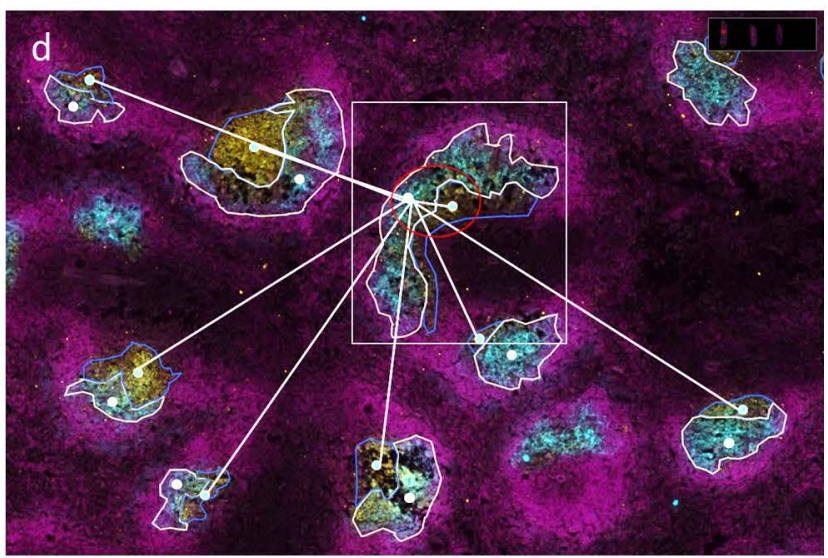

Germinal Center Light and Dark Zone Pair Detection

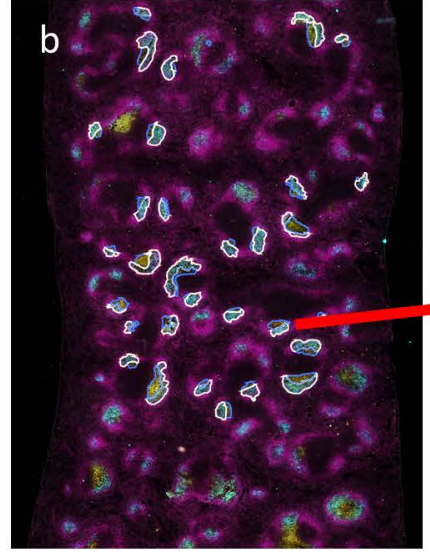

Mouse Spleen IF Annotated

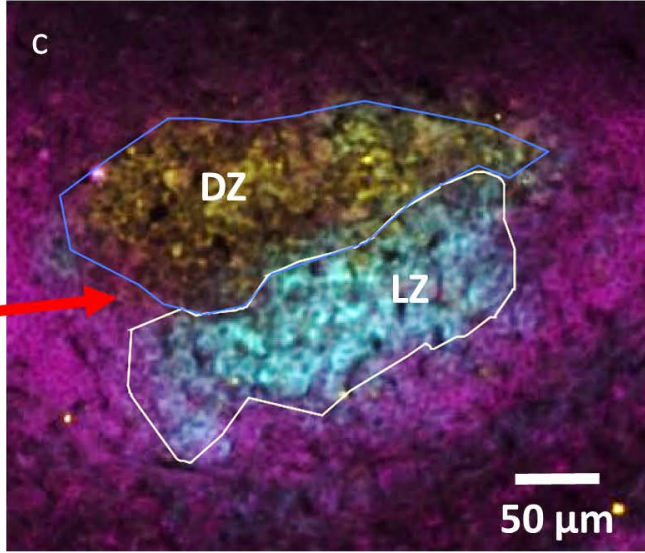

Mouse Spleen IF Annotated Zoom

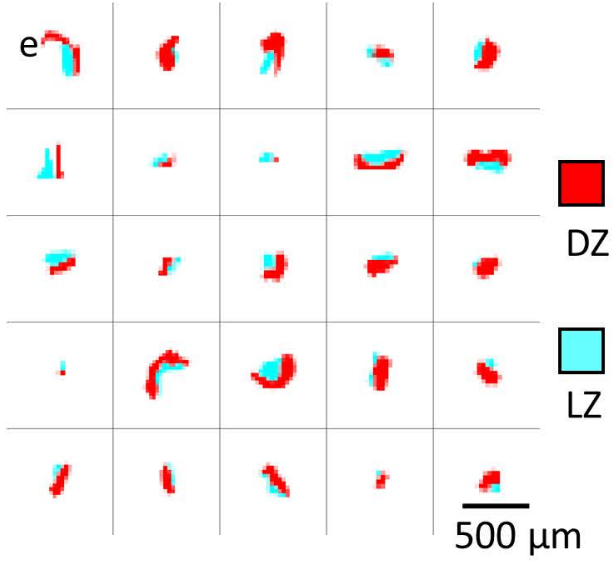

Data Extraction and Statistical Analysis 


\section{Supplemental Figure 4}

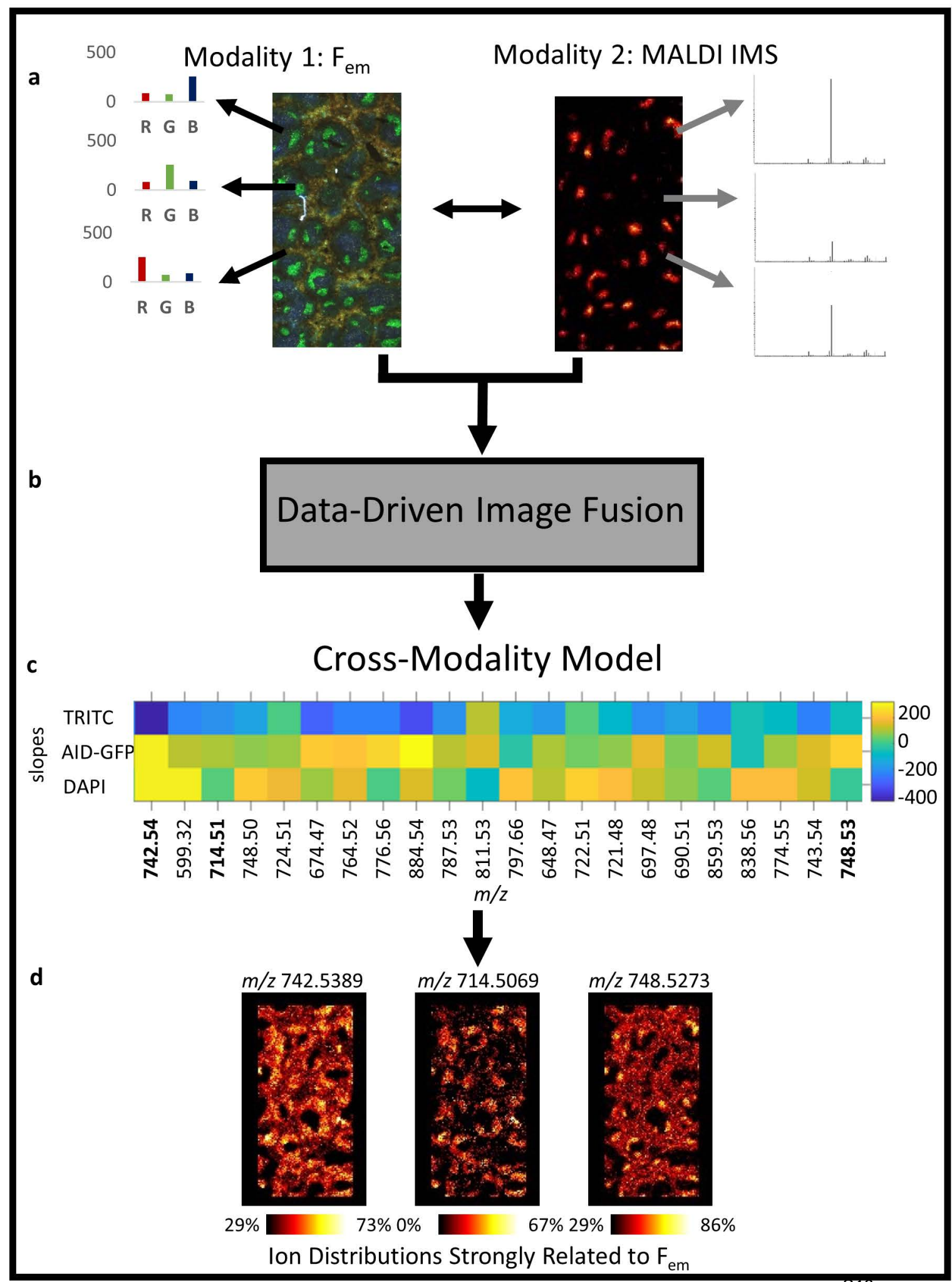




\section{Supplemental Figure 5}

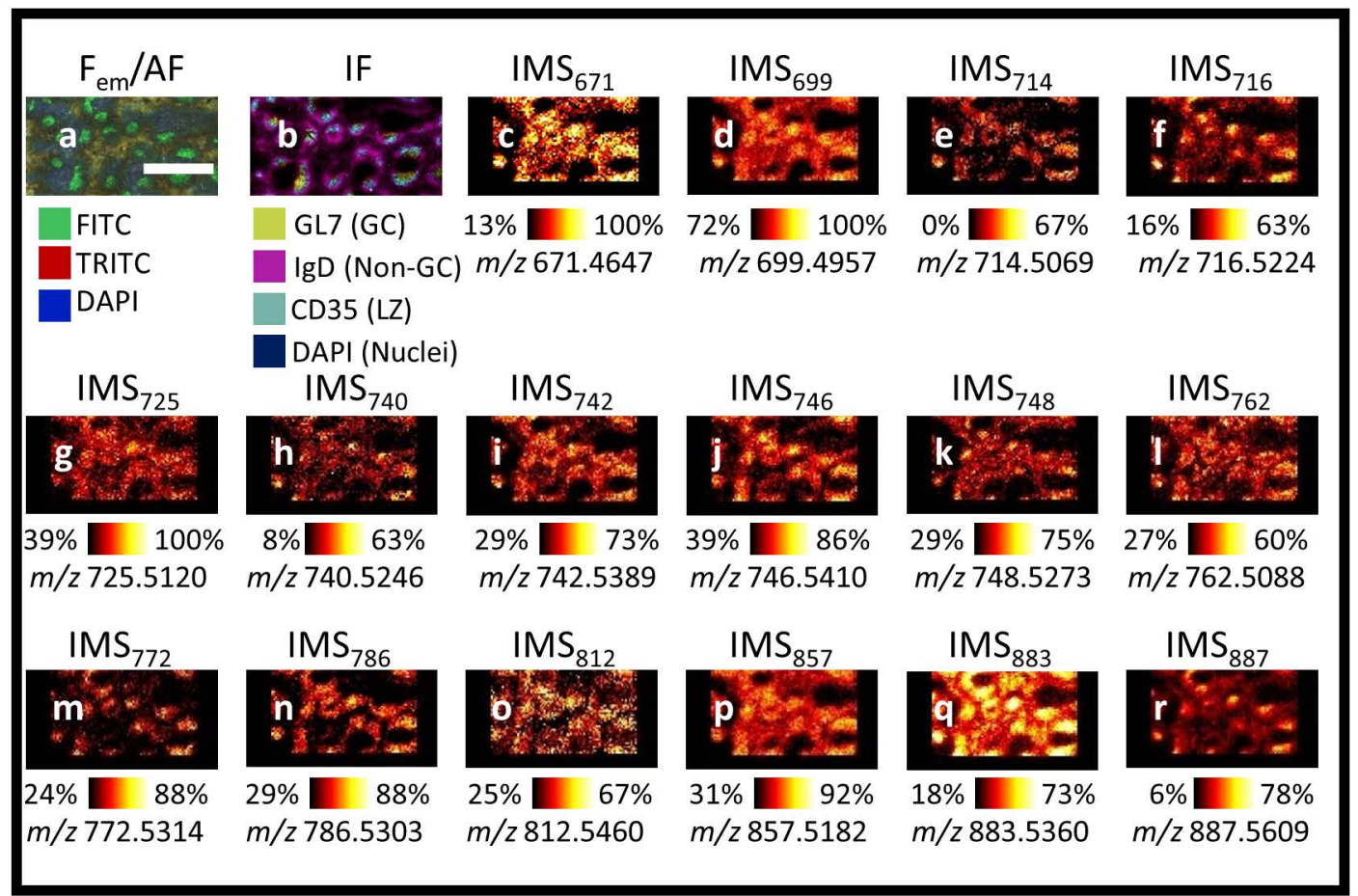


File Edit View Analysis Tools Compass Help

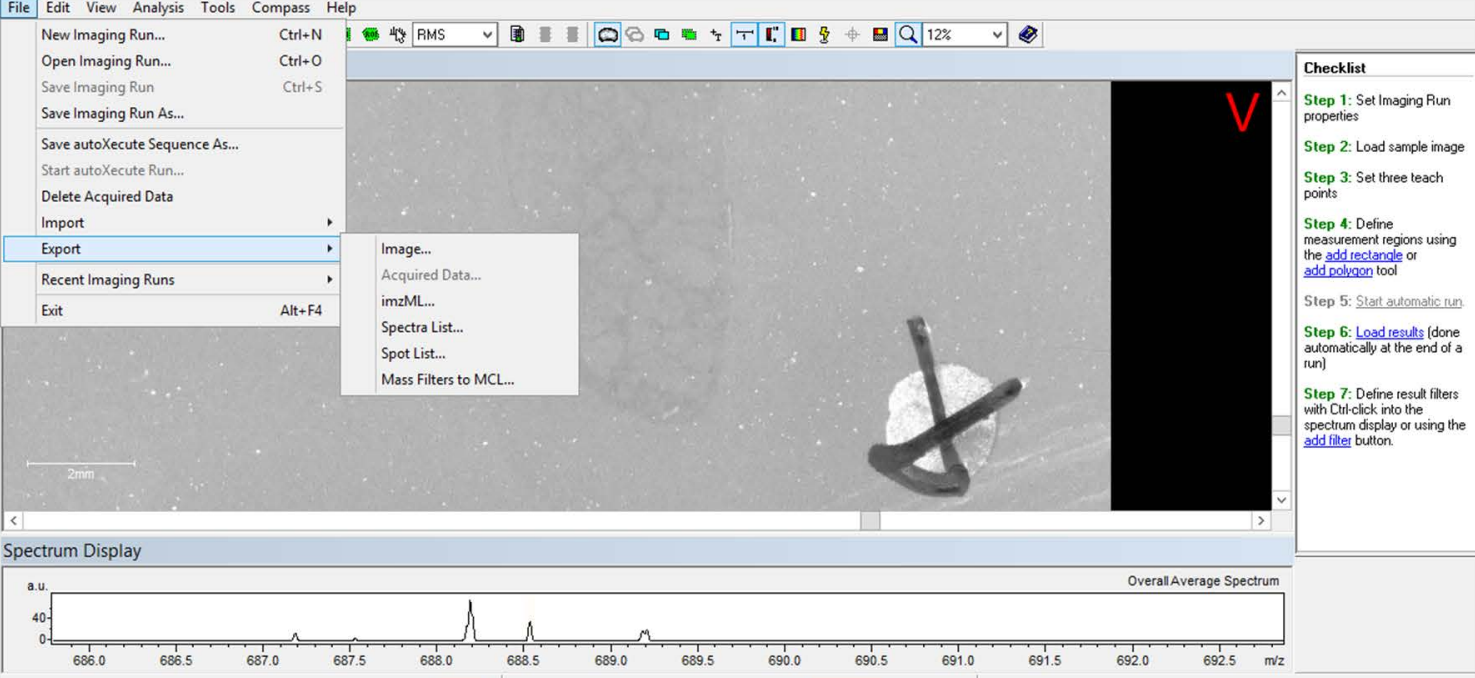

1 Measurement Region(s), 1558 Postion(s)

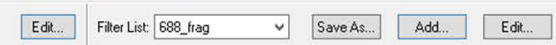

\begin{tabular}{|c|c|c|c|c|c|c|c|}
\hline$\square$ Region & Raster (um) & Acquisition Method & $\square$ Spectrum & $\square \square$ Filter Name & Filler Method , & Hits & $\Lambda$ \\
\hline \multirow[t]{4}{*}{$\square 01$} & 120,120 & D:MethodsiMS_M. & $\square$ & 03 & $168.0395 \mathrm{Da} \pm 0.02 \mathrm{Da}$ & 1026 & \\
\hline & & & & 04 & $255.2312 \mathrm{Da} \pm 0.02 \mathrm{Da}$ & 1247 & $\equiv$ \\
\hline & & & & 02 & $281.2452 \mathrm{Da} \pm 0.02 \mathrm{Da}$ & 1119 & \\
\hline & & & & 05 & $283.2595 \mathrm{Da} \pm 0.02 \mathrm{Da}$ & 919 & $v$ \\
\hline
\end{tabular}

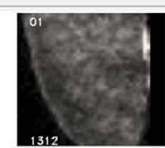

$688.5383 \mathrm{Da} \pm 0.02 \mathrm{Da}$

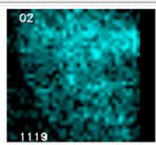

$281.2452 \mathrm{Da}=0.02 \mathrm{Da}$

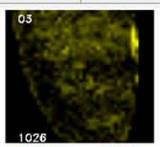

$168.0395 \mathrm{Da} \pm 0.02 \mathrm{Da}$

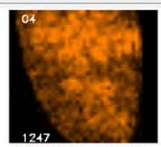

$255.2312 \mathrm{Da}=0.02 \mathrm{Da}$ b

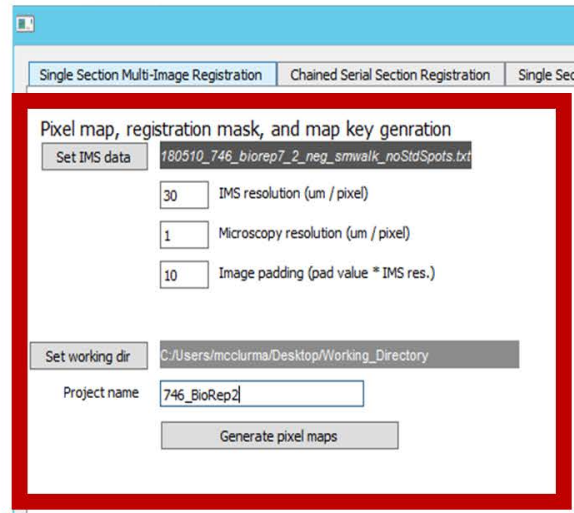

MSRC Autofluorescence Registration Toolbox

Coordinate extraction from Image] ROIs

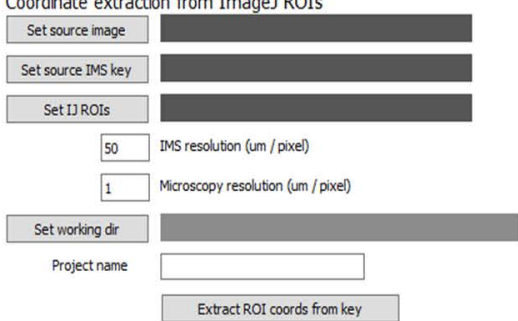


Supplemental Figure 7

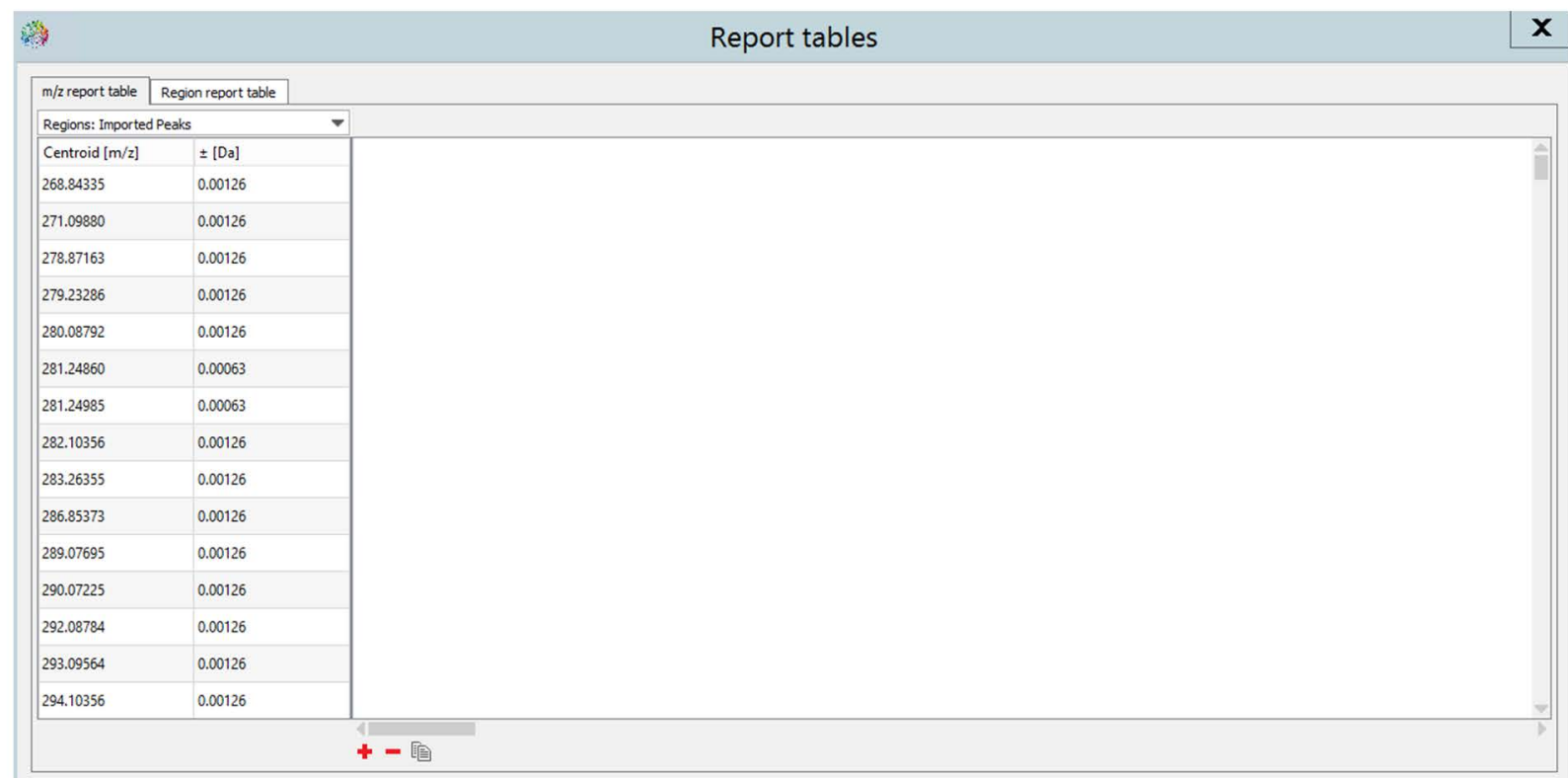




\section{Supplemental Figure 8}

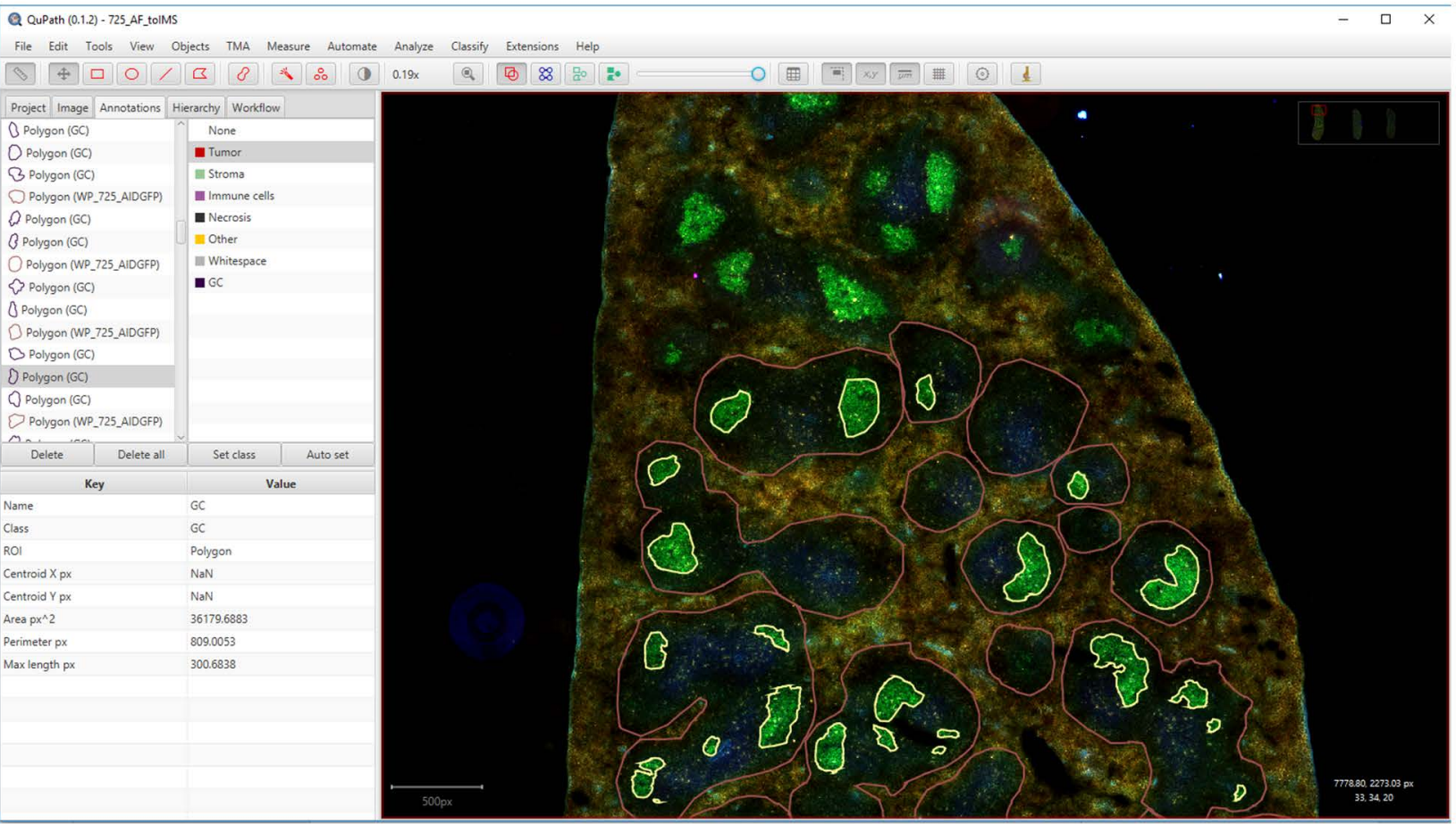




\section{Supplemental Figure 9}

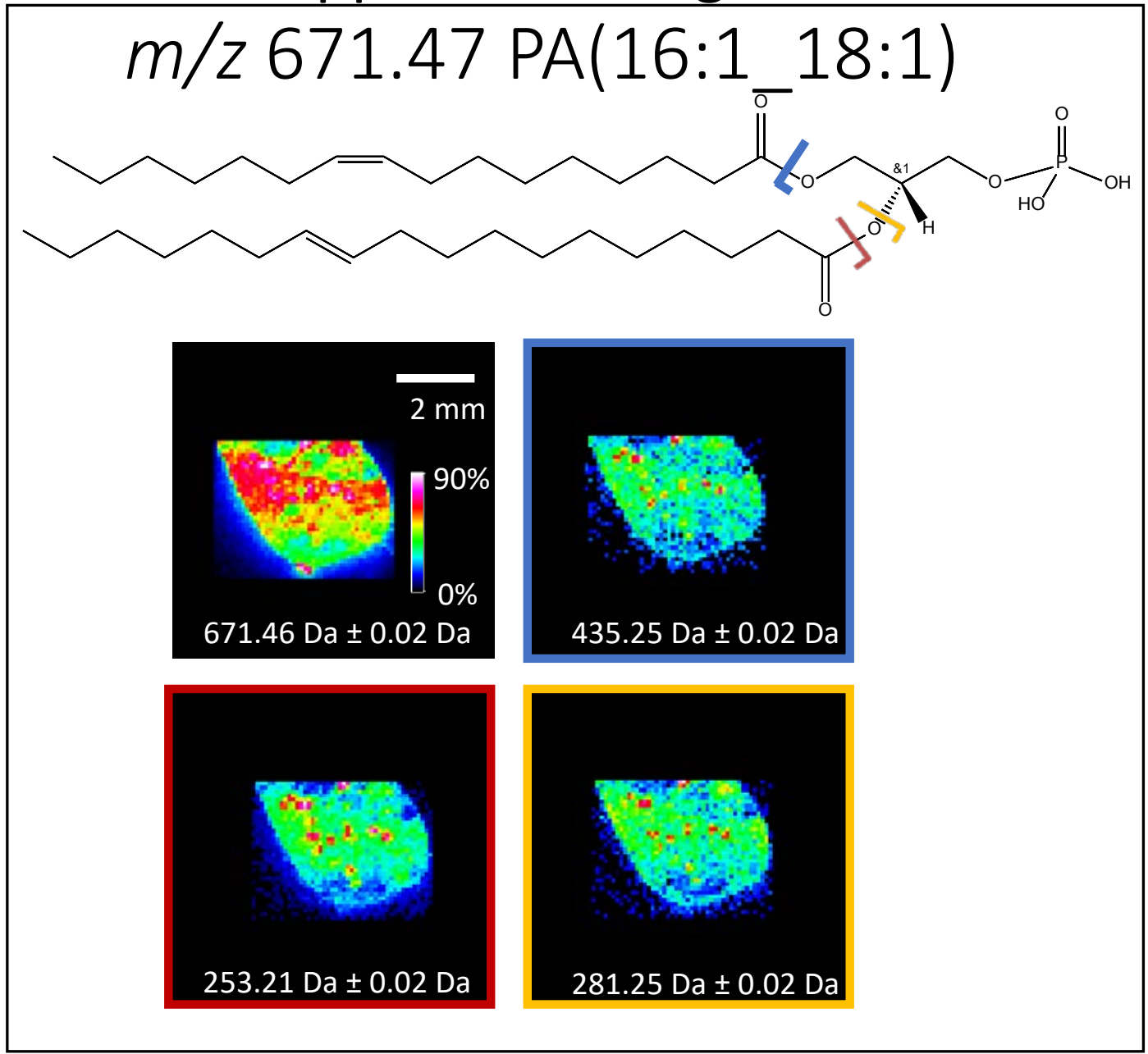


Supplemental Figure 10

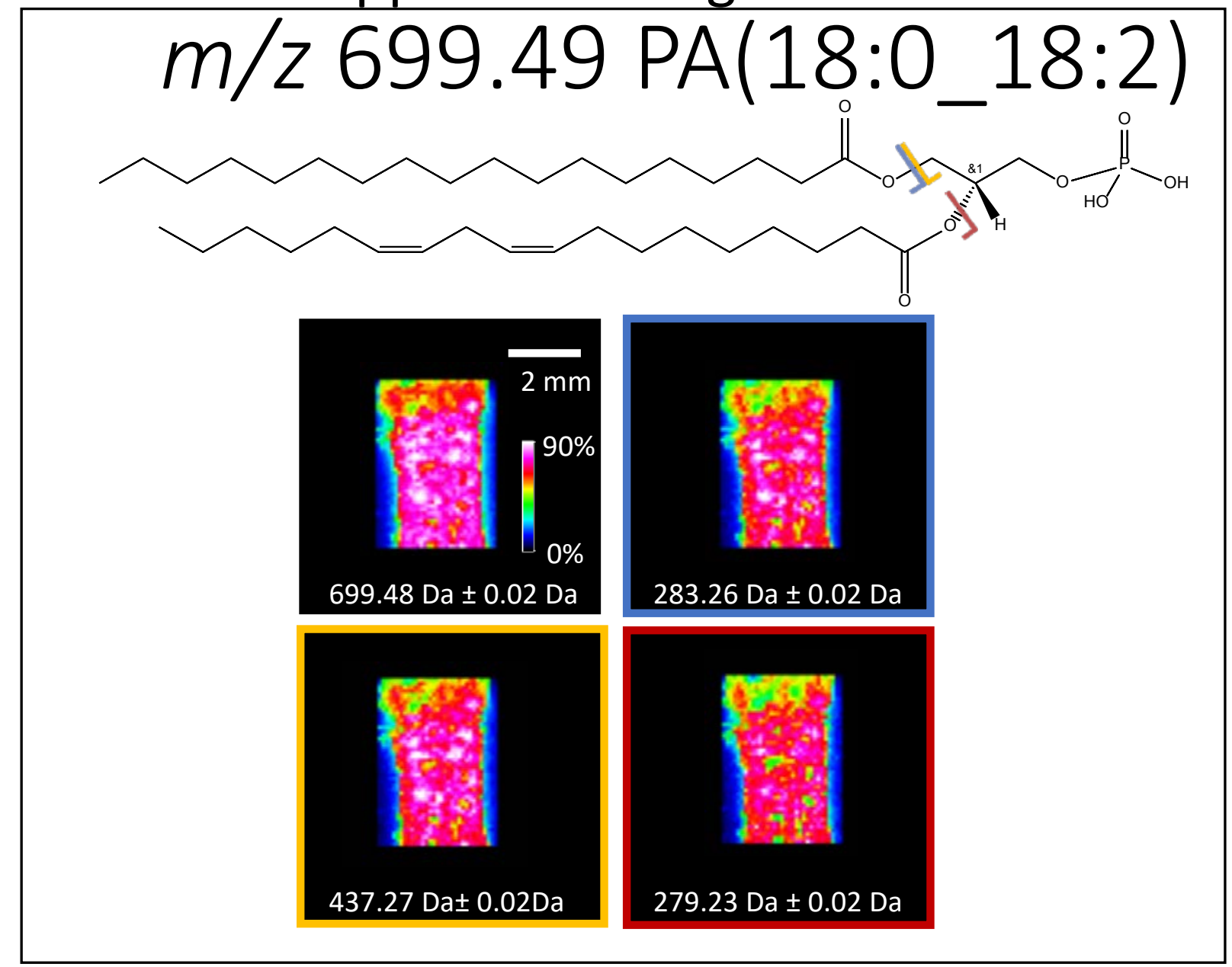


Supplemental Figure 11

$$
\text { m/z 699.49 PA(16:0_20:2) }
$$
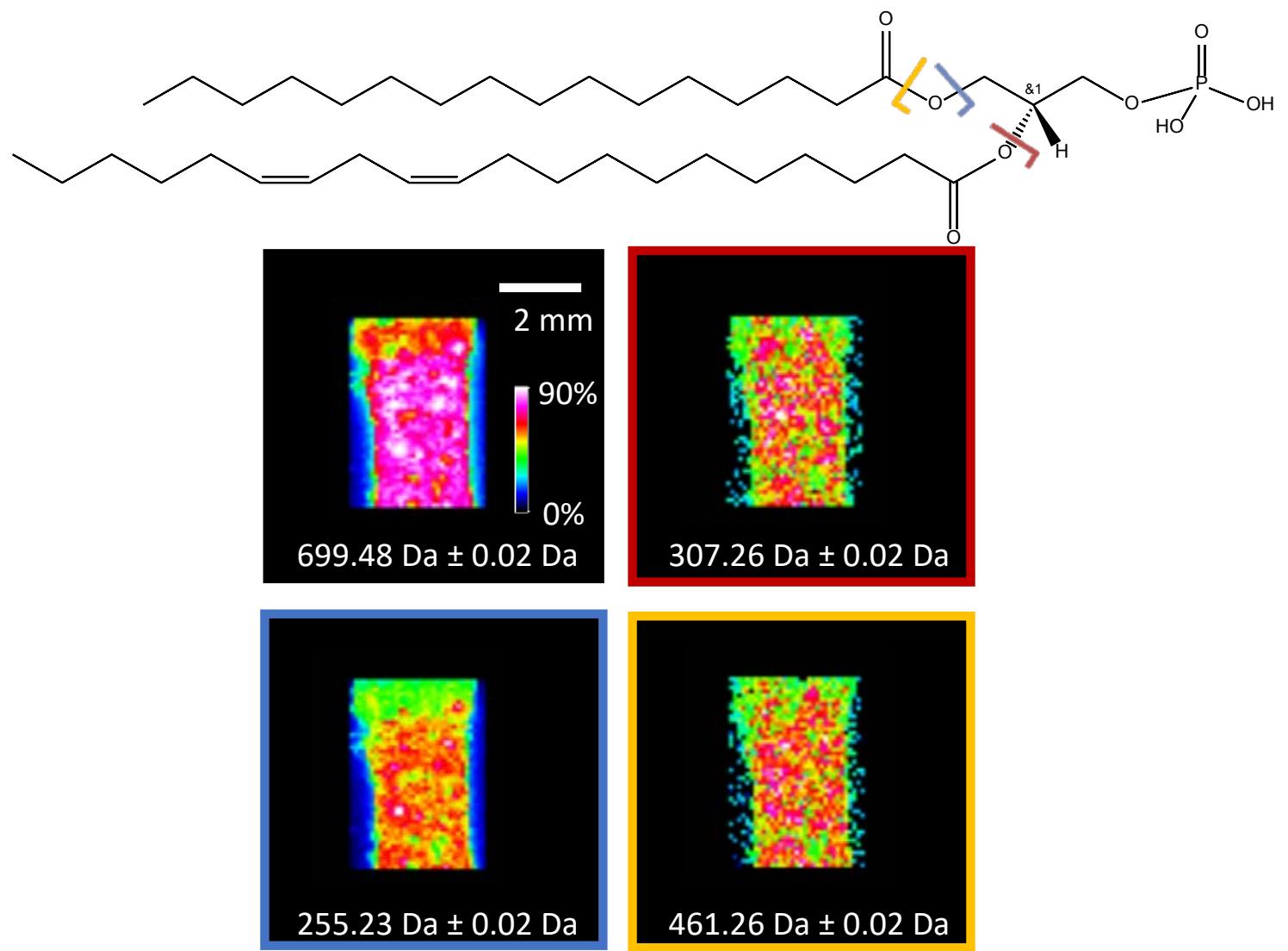
Supplemental Figure 12

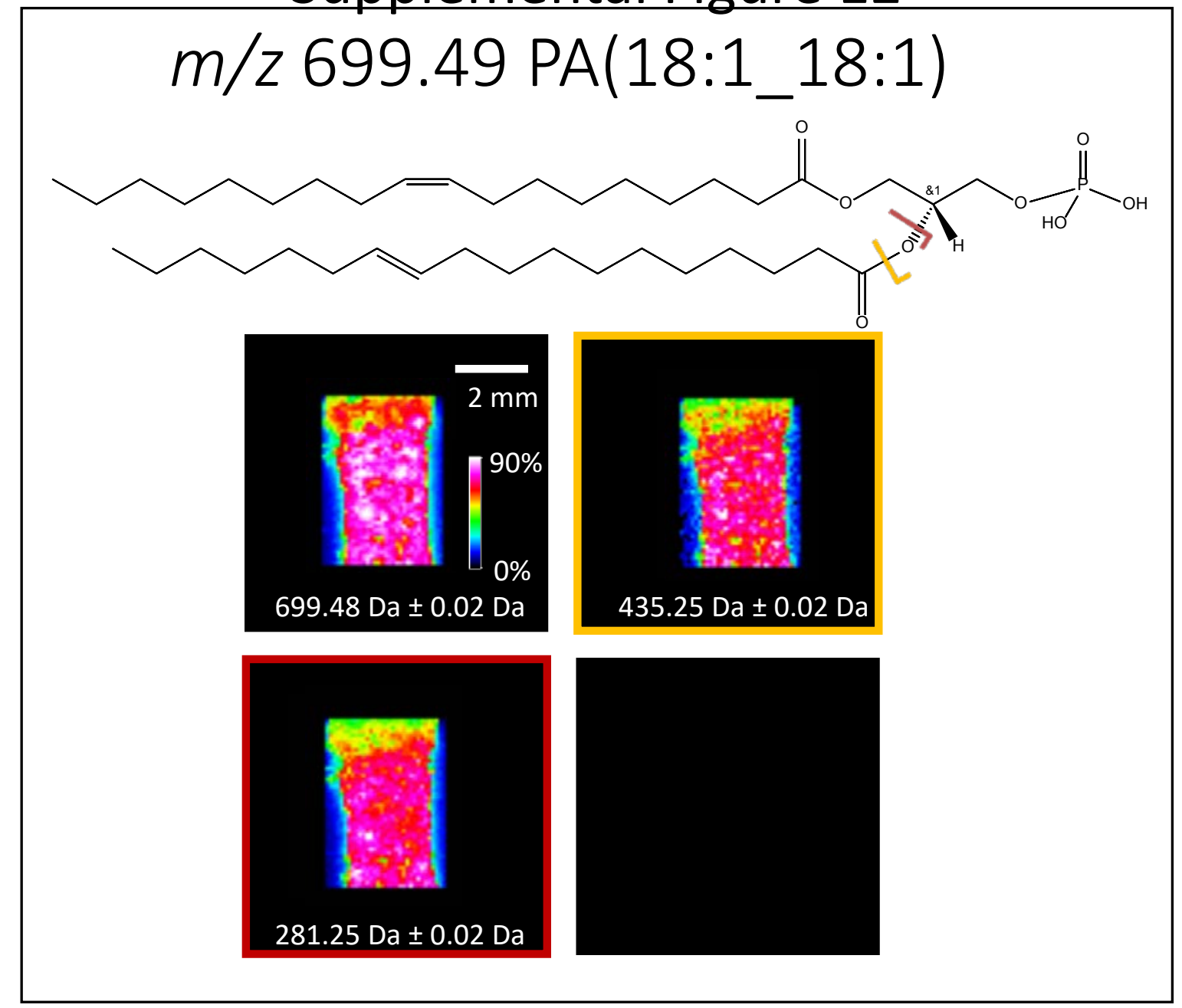




\section{Supplemental Figure 13}

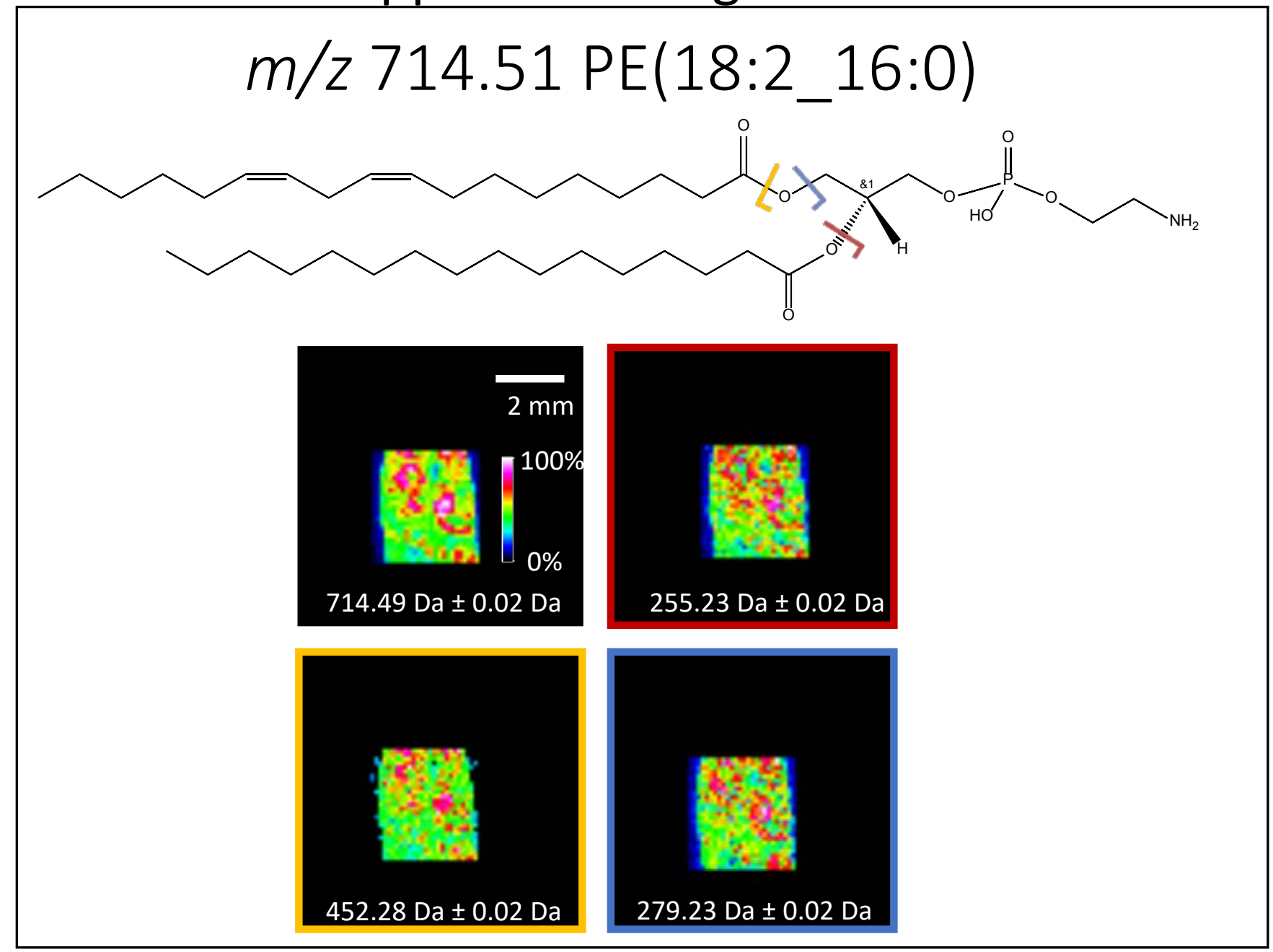




\section{Supplemental Figure 14}

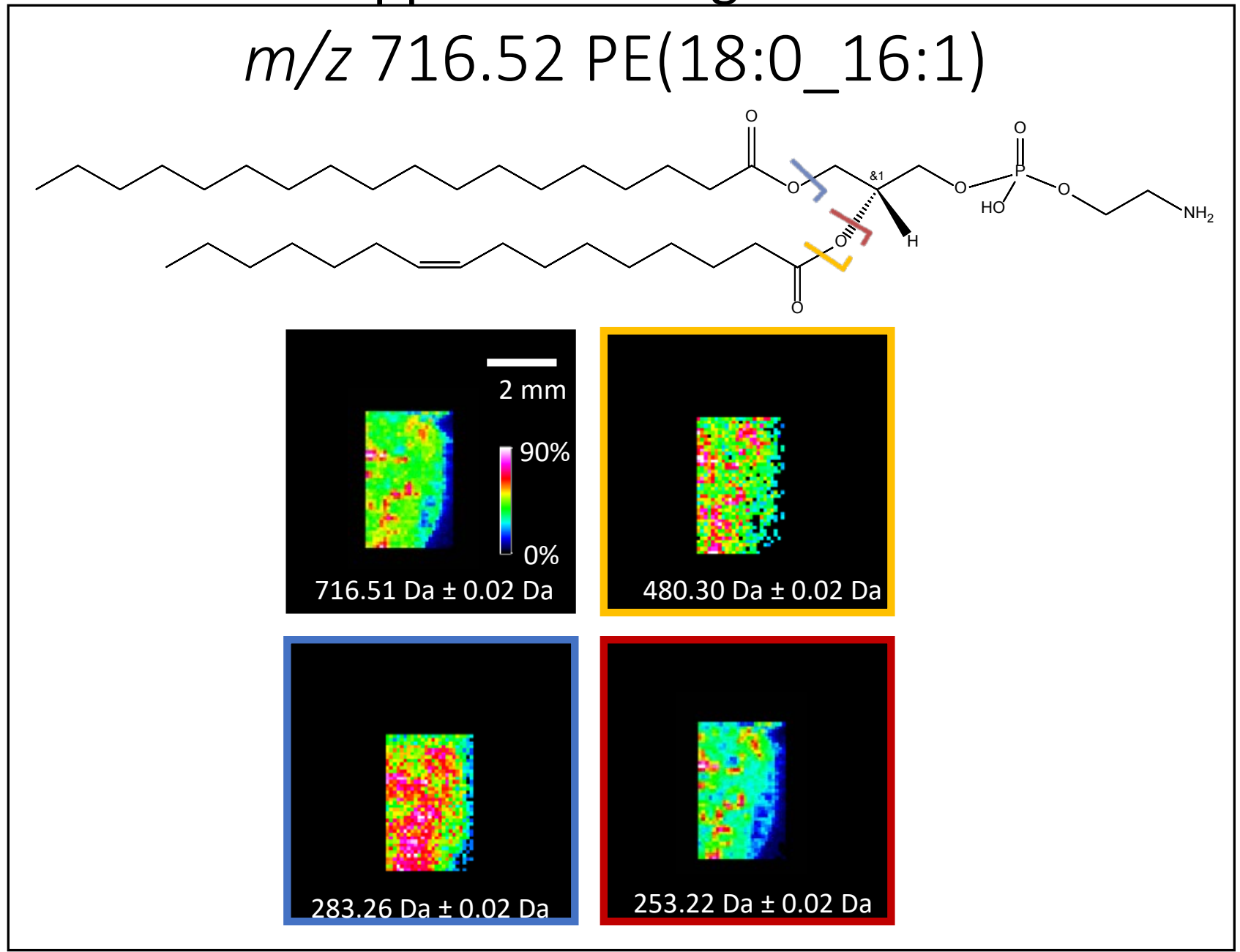


Supplemental Figure 15

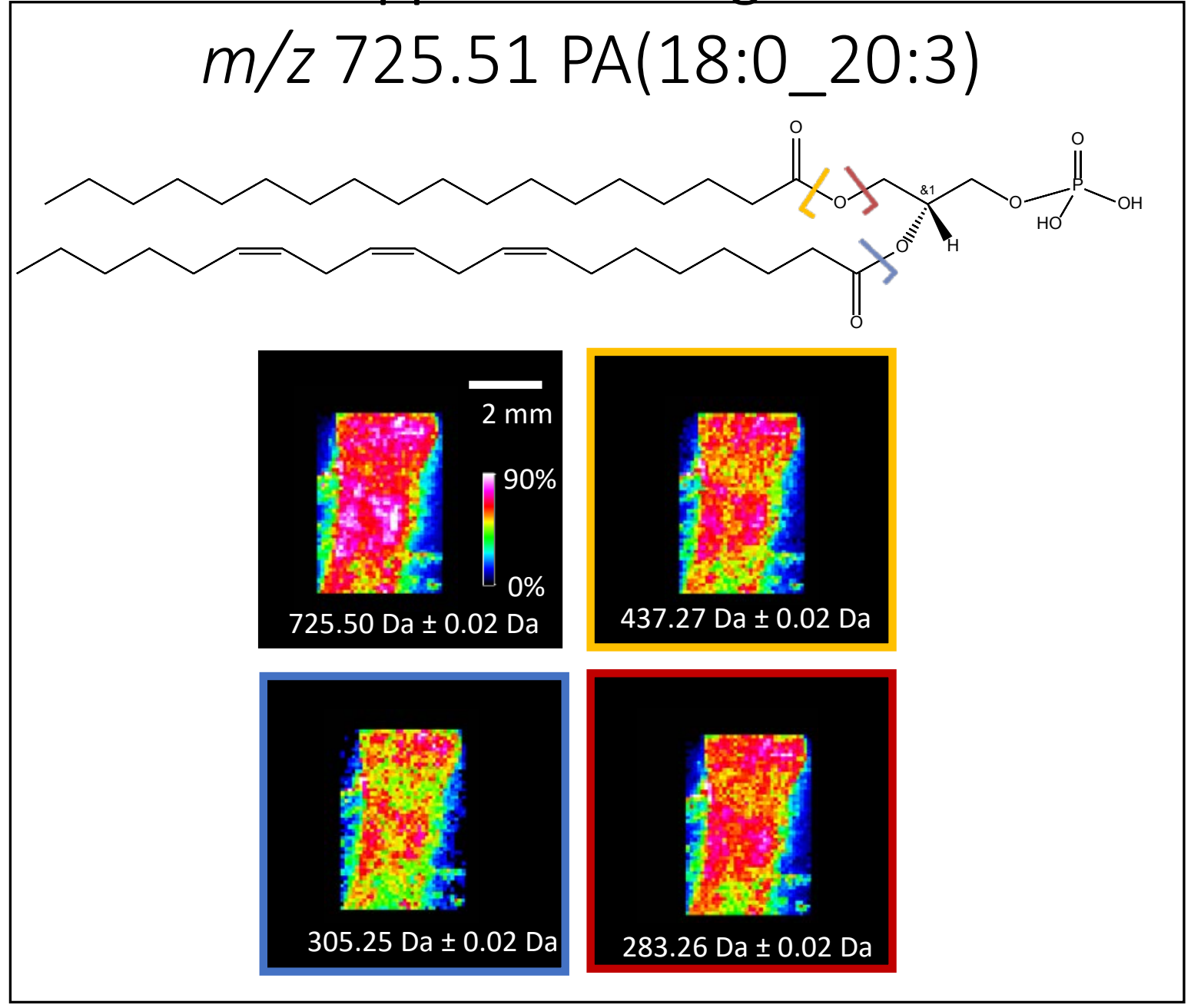


Supplemental Figure 16

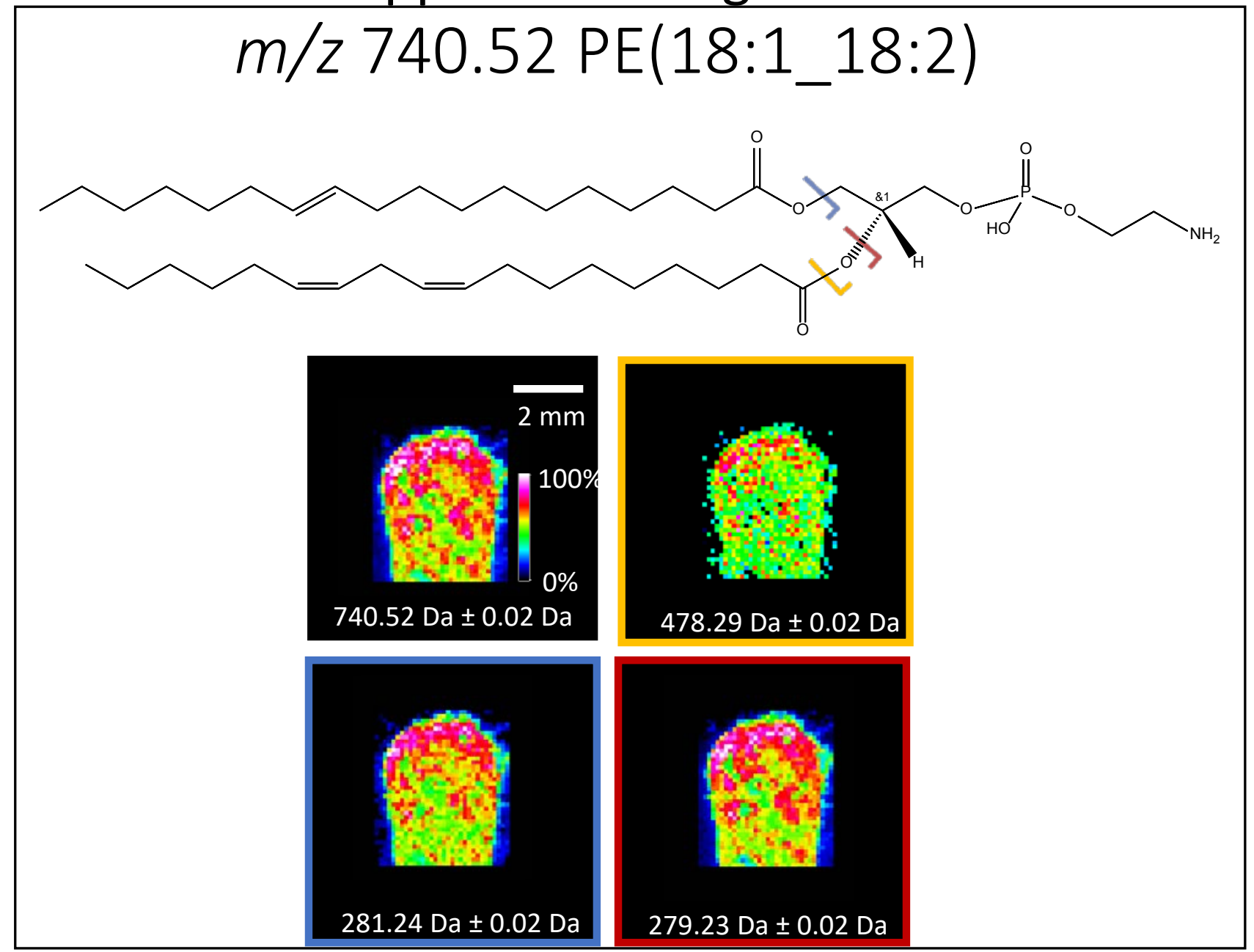


Supplemental Figure 17

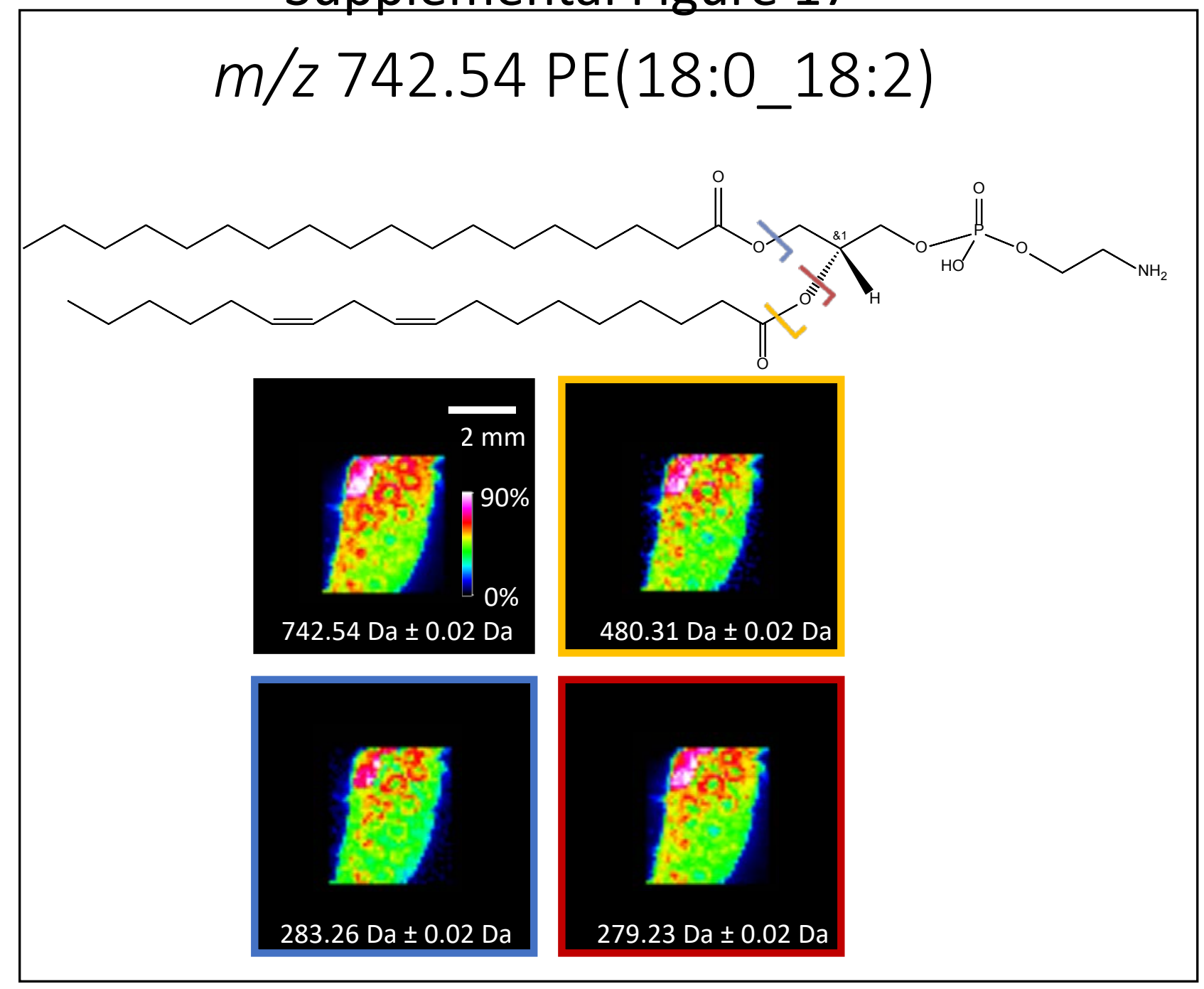




\section{Supplemental Figure 19}

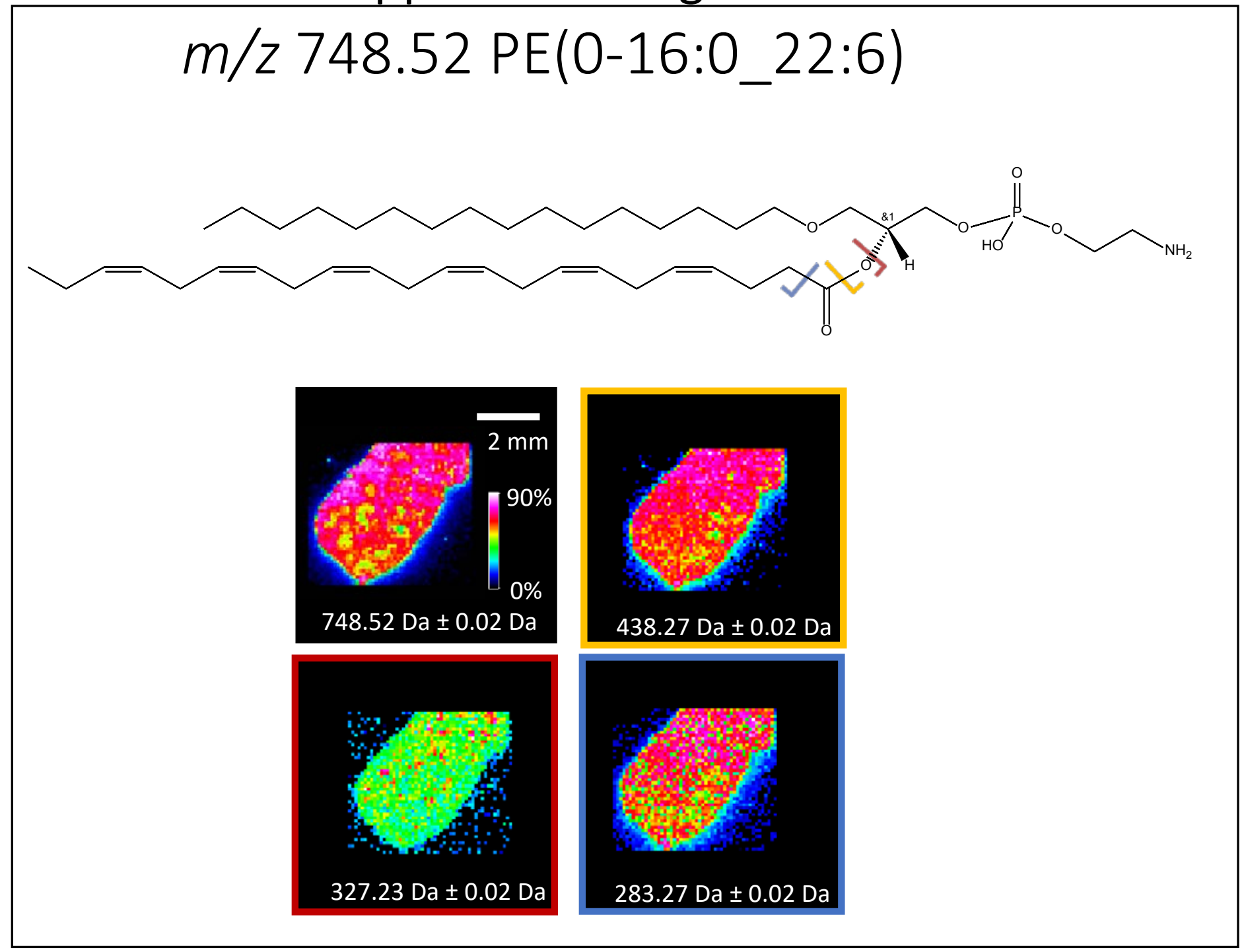




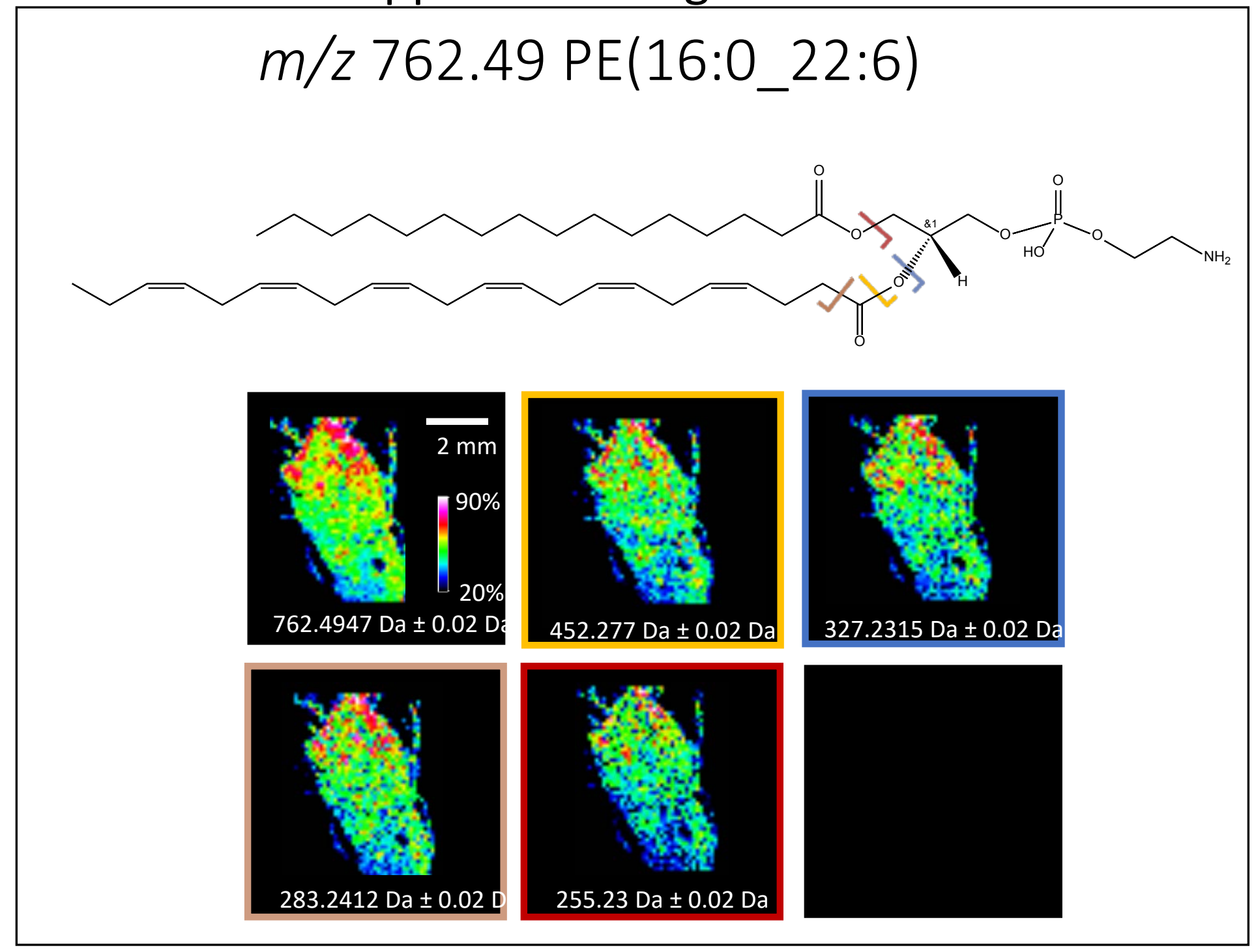


Supplemental Figure 21

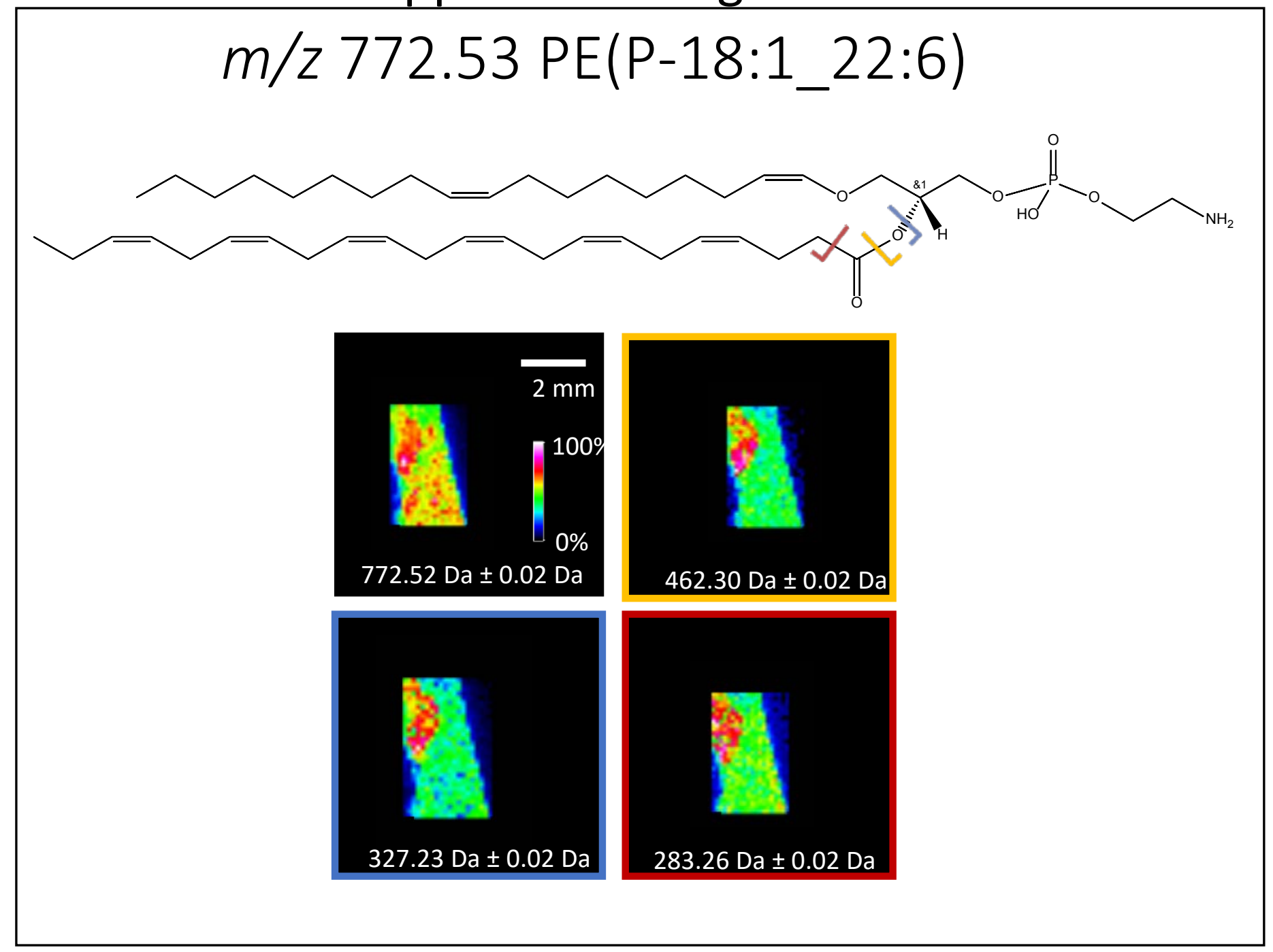




\section{Supplemental Figure 22}

\section{m/z 786.52 PS(18:0_18:2)}
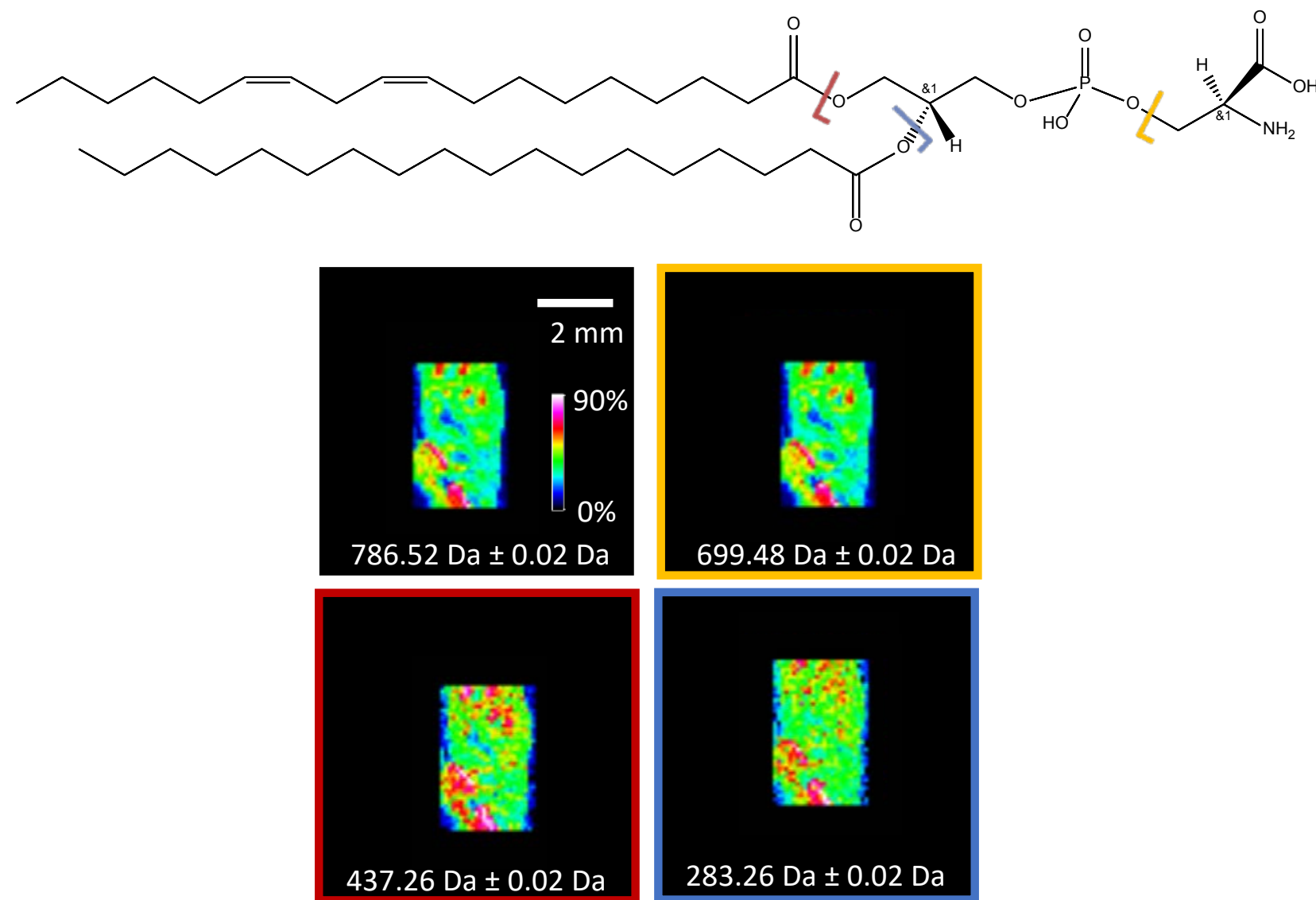
Supplemental Figure 23

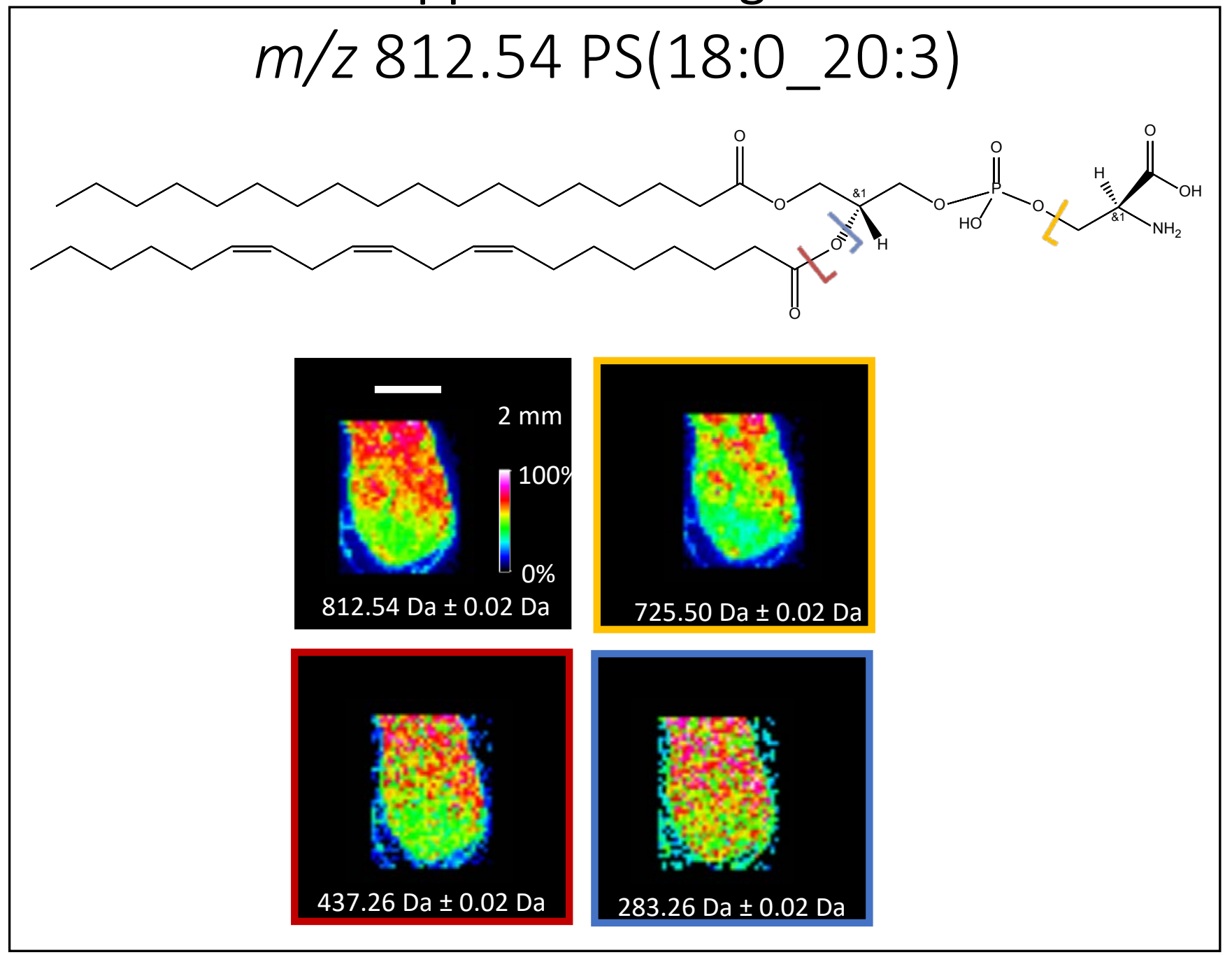




\section{Supplemental Figure 24}

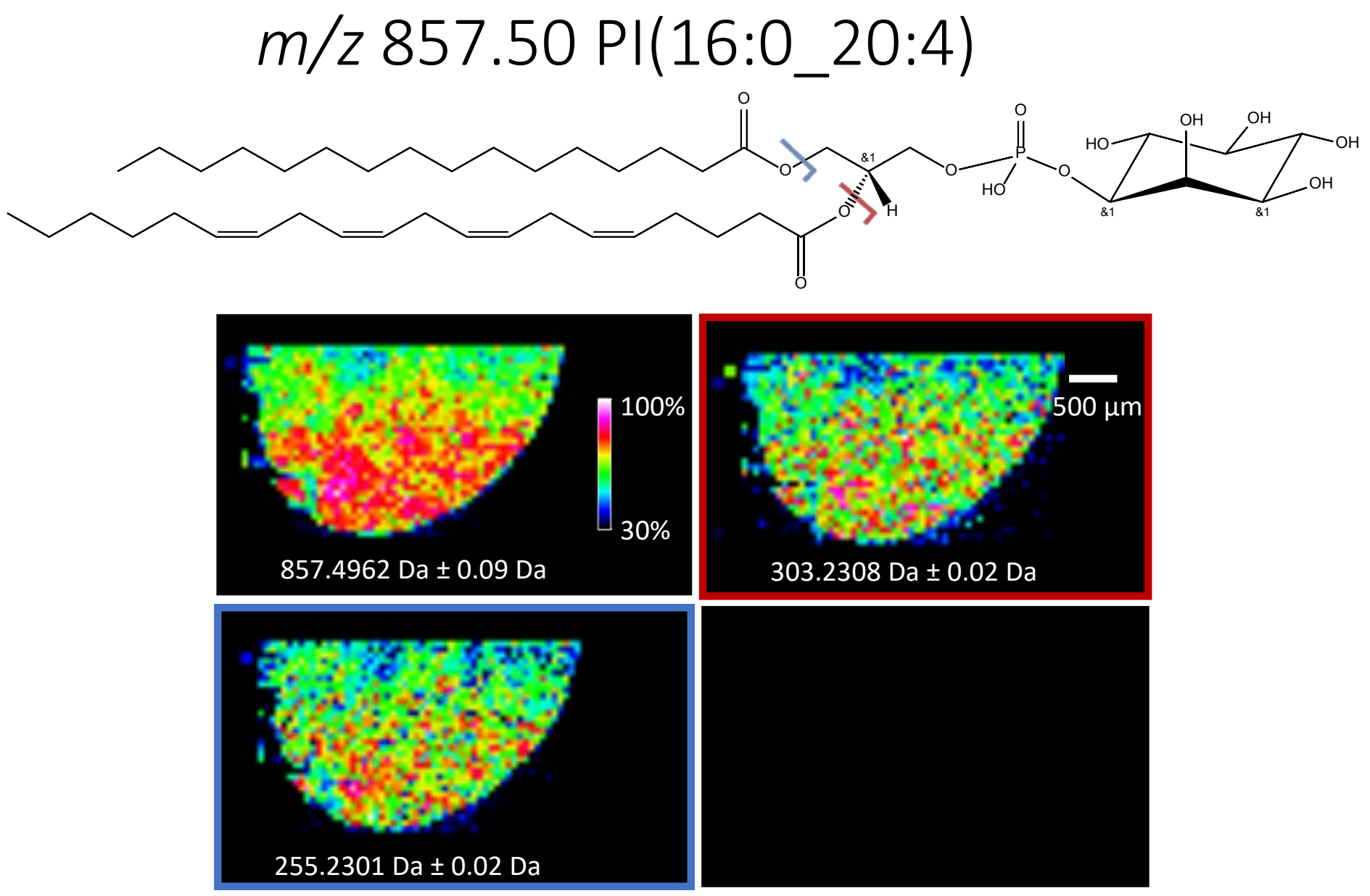




\section{Supplemental Figure 25}

\section{m/z 883.53 PI(18:1_20:4)}
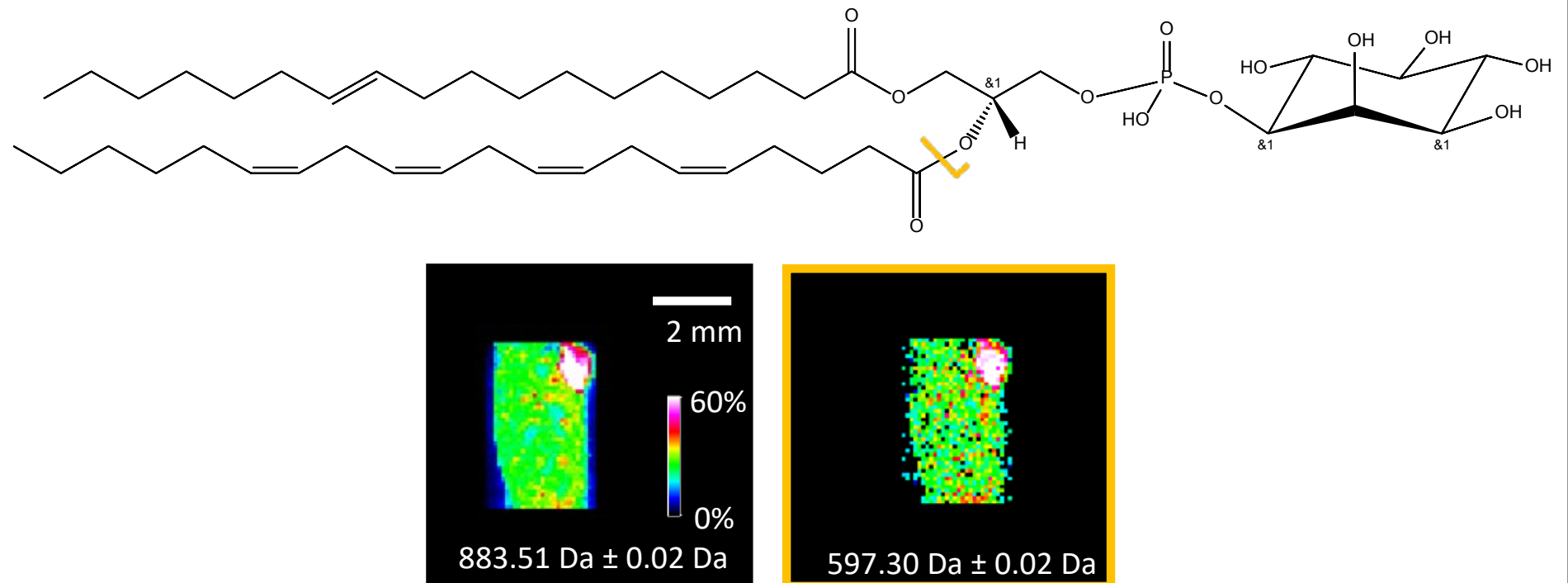
Supplemental Figure 26

\section{m/z 887.56 PI(18:0_20:3)}
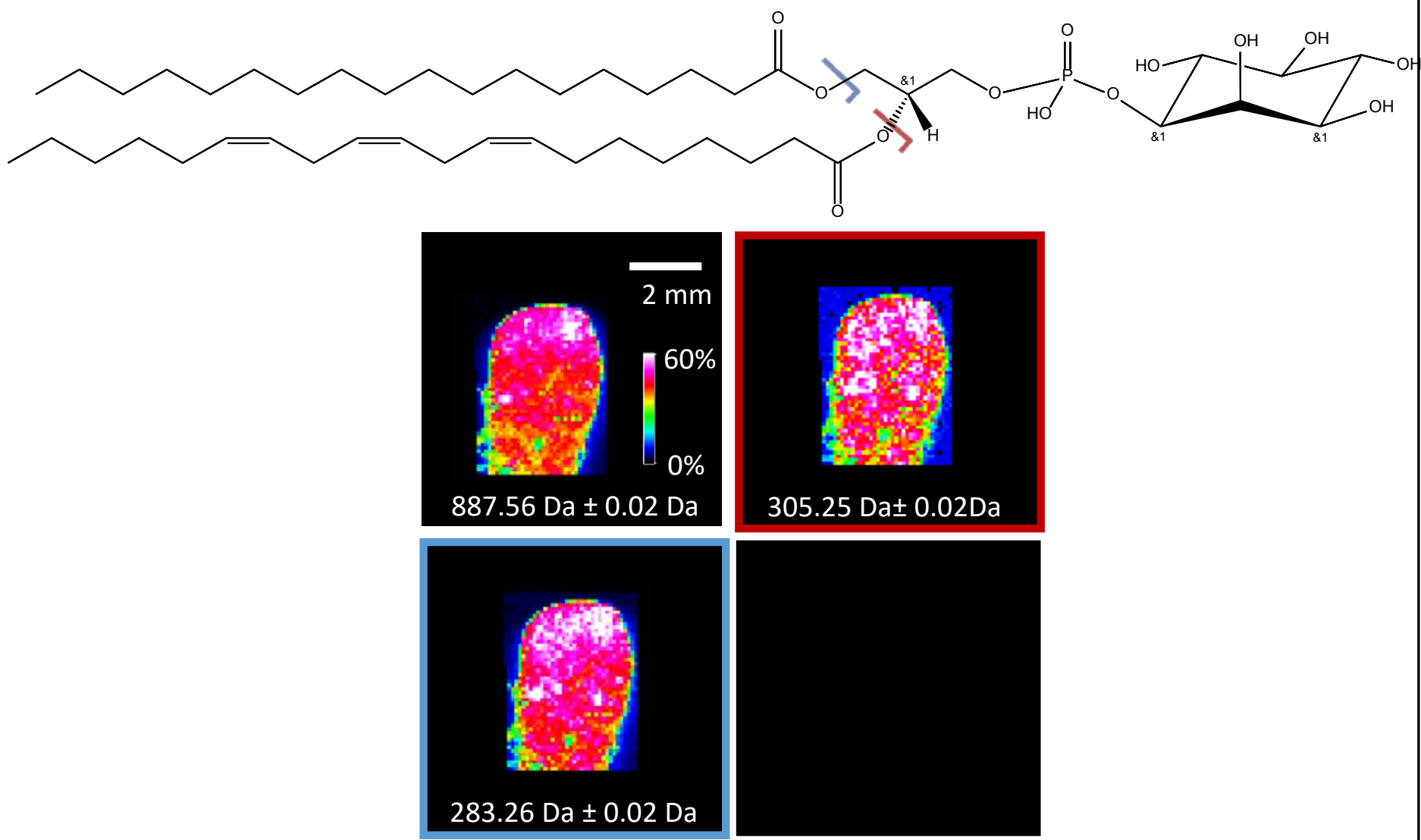
Supplemental Figure 27

\section{m/z 788.52 PS(18:1_18:0)}

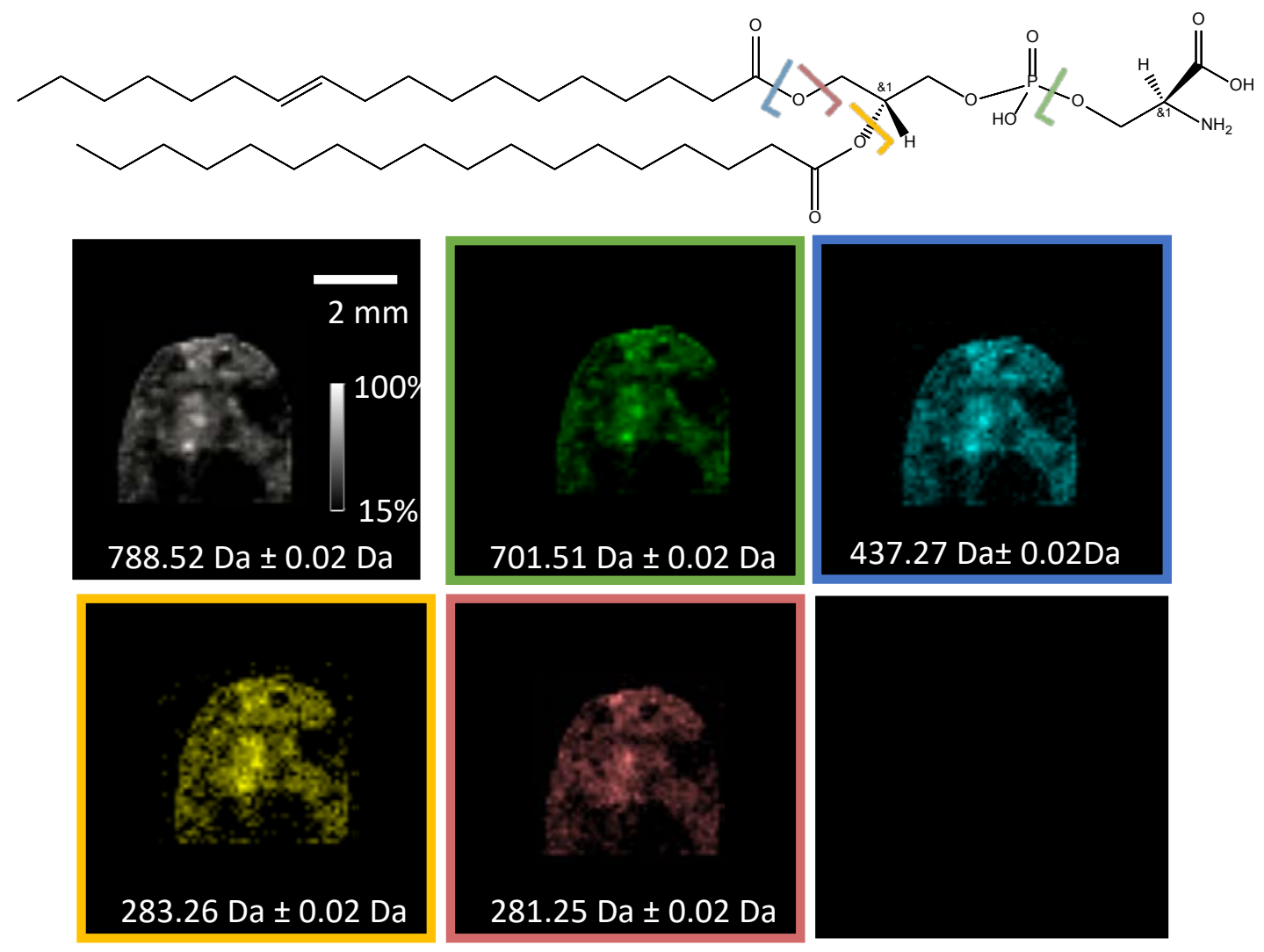


Supplemental Table 1: Serial section comparison. For each serial section a Dice Coefficient was determined and the mean and standard deviation are shown.

\begin{tabular}{|c|c|}
\hline Sample & Dice Coefficient \\
\hline Serial HE, 725 & 0.83 \\
\hline Serial IHC, 725 & 0.85 \\
\hline Serial IHC, 745 & 0.80 \\
\hline Serial HE, 737 & 0.79 \\
\hline Serial IHC, 737 & 0.78 \\
\hline mean dice & 0.81 \\
\hline st. dev. Dice & 0.029 \\
\hline
\end{tabular}


Supplemental Table 2: Segmentation determined ions of interest. For each biological replicate, the $\mathrm{m} / \mathrm{z}$ value and tstatistic output from spatially shrunken segmentation in Cardinal is shown.

\begin{tabular}{|r|r|r|r|r|r|}
\hline \multicolumn{2}{|c|}{ Biological Rep 1 } & \multicolumn{2}{c|}{ Biological Rep 2 } & \multicolumn{2}{c|}{ Biological Rep 3 } \\
\hline$m / z$ & tstatistics & \multicolumn{1}{c|}{$m / z$} & tstatistics & \multicolumn{1}{c|}{$m / z$} & tstatistics \\
\hline 871.5659 & 34.48 & 883.5369 & 42.26 & 871.5659 & 68.09 \\
\hline 883.5369 & 33.13 & 857.5195 & 37.10 & 888.5639 & 64.87 \\
\hline 1626.9414 & 32.19 & 871.5659 & 36.96 & 887.5639 & 61.83 \\
\hline 1642.9175 & 31.93 & 1642.9175 & 36.28 & 1532.8099 & 61.47 \\
\hline 1627.9478 & 31.63 & 1643.9474 & 34.70 & 1626.9414 & 61.25 \\
\hline 752.5599 & 31.15 & 869.5500 & 33.15 & 1627.9478 & 59.01 \\
\hline 1643.9474 & 30.84 & 752.5599 & 32.70 & 752.5599 & 55.64 \\
\hline 857.5195 & 29.02 & 776.5584 & 31.81 & 883.5369 & 55.53 \\
\hline 884.5392 & 28.81 & 1644.9251 & 30.47 & 1628.9590 & 53.42 \\
\hline 869.5500 & 28.23 & 884.5392 & 30.08 & 1533.8367 & 53.34 \\
\hline 1532.8099 & 27.20 & 858.5210 & 28.18 & 1642.9175 & 53.27 \\
\hline 887.5639 & 27.15 & 887.5639 & 27.69 & 1643.9474 & 51.60 \\
\hline 776.5584 & 26.93 & 746.5143 & 27.57 & 872.5693 & 50.55 \\
\hline 1641.9245 & 26.18 & 859.5297 & 26.23 & 760.5078 & 49.13 \\
\hline 1517.8449 & 25.80 & 786.5288 & 25.55 & 776.5584 & 48.90 \\
\hline 1533.8367 & 25.48 & 1641.9245 & 24.73 & 1517.8449 & 48.54 \\
\hline 1644.9251 & 25.01 & 1626.9414 & 24.70 & 1644.9251 & 47.54 \\
\hline 858.5210 & 23.43 & 835.5270 & 23.28 & 884.5392 & 46.99 \\
\hline 1628.9590 & 22.27 & 860.5320 & 22.59 & 716.5190 & 46.56 \\
\hline 888.5639 & 21.91 & 885.5499 & 22.33 & 753.5596 & 45.58 \\
\hline
\end{tabular}


Supplemental Table 3. Image Fusion Results. Because image fusion is based on a linear regression model, the relation of ions to a color channel can be drawn from the slope value of the correlation function. From left to right the $\mathrm{m} / \mathrm{z}$ value, the red channel slope value, the green channel slope value, and the blue channel slope value are shown. The higher positive number the greater the correlation and vice versa.

\begin{tabular}{|l|r|r|r|}
\hline$m / z$ & \multicolumn{1}{l|}{$\begin{array}{l}\text { Red } \\
\text { Channel }\end{array}$} & \multicolumn{1}{l|}{$\begin{array}{l}\text { Green } \\
\text { Channel }\end{array}$} & \multicolumn{1}{l|}{$\begin{array}{l}\text { Blue } \\
\text { Channel }\end{array}$} \\
\hline 885.5506 & -2910.1 & 1647.6 & 6863.1 \\
\hline 886.5552 & -1413.0 & 814.4 & 3347.6 \\
\hline 883.5354 & -587.4 & 565.8 & 257.8 \\
\hline 857.5190 & -582.6 & 400.6 & 351.3 \\
\hline 718.5403 & -575.3 & 356.6 & 422.3 \\
\hline 885.5377 & -546.0 & 345.8 & 1313.9 \\
\hline 884.5386 & -345.4 & 321.7 & 142.0 \\
\hline 673.4820 & -467.9 & 316.7 & 363.3 \\
\hline 742.5399 & -445.2 & 290.5 & 288.2 \\
\hline 746.5143 & -394.6 & 280.0 & 218.4 \\
\hline 786.5290 & -507.4 & 279.4 & 140.8 \\
\hline 699.4981 & -457.5 & 277.2 & 295.9 \\
\hline 810.5302 & 212.1 & 273.6 & -91.5 \\
\hline 887.5686 & -579.2 & 252.9 & 419.7 \\
\hline 647.4662 & -406.1 & 246.1 & 255.4 \\
\hline 776.5604 & -237.7 & 244.7 & 22.1 \\
\hline 861.5505 & -357.8 & 244.6 & 100.5 \\
\hline 716.5246 & -457.9 & 243.8 & 198.7 \\
\hline 748.5301 & -69.7 & 236.6 & -12.2 \\
\hline 835.5335 & -384.3 & 236.5 & 243.8 \\
\hline 834.5304 & -341.0 & 222.2 & 419.1 \\
\hline 671.4660 & -292.4 & 222.1 & 86.0 \\
\hline 752.5608 & -277.4 & 219.0 & 12.2 \\
\hline 747.5174 & -398.2 & 211.3 & 240.2 \\
\hline 887.5556 & -350.4 & 209.4 & 883.1 \\
\hline 858.5234 & -323.4 & 207.0 & 199.8 \\
\hline 764.5243 & -229.8 & 203.0 & 155.8 \\
\hline
\end{tabular}




\begin{tabular}{|r|r|r|r|}
\hline 723.4982 & 28.7 & 201.2 & 334.9 \\
\hline 886.5406 & -301.3 & 183.7 & 726.7 \\
\hline 744.5561 & -314.8 & 176.1 & 462.3 \\
\hline 719.5433 & -288.2 & 167.3 & 219.5 \\
\hline 762.5090 & -362.2 & 167.0 & 319.6 \\
\hline 772.5290 & -139.9 & 163.3 & 25.7 \\
\hline 697.4823 & -214.9 & 157.9 & 129.6 \\
\hline 747.4978 & -286.4 & 157.6 & 426.4 \\
\hline 674.4851 & -230.6 & 150.8 & 160.5 \\
\hline 811.5335 & 112.8 & 149.9 & -81.5 \\
\hline 743.5426 & -238.8 & 147.7 & 140.9 \\
\hline 888.5696 & -320.9 & 139.4 & 230.8 \\
\hline 788.5245 & -149.2 & 133.4 & 74.6 \\
\hline 859.5346 & -240.0 & 130.1 & 24.6 \\
\hline 700.5013 & -222.8 & 124.8 & 123.2 \\
\hline 645.4505 & -213.7 & 117.5 & 45.7 \\
\hline 599.3213 & -223.4 & 114.9 & 286.4 \\
\hline 862.5535 & -180.6 & 113.2 & 47.6 \\
\hline 740.5244 & -153.0 & 112.8 & 28.0 \\
\hline 749.5317 & -39.7 & 112.1 & -1.2 \\
\hline 717.5273 & -197.6 & 109.8 & 59.5 \\
\hline 787.5325 & -202.5 & 109.0 & 47.9 \\
\hline 714.5086 & -196.2 & 102.5 & 26.4 \\
\hline 738.5086 & -244.3 & 98.5 & 222.2 \\
\hline 765.5280 & -125.4 & 95.2 & 83.9 \\
\hline 648.4696 & -178.2 & 94.9 & 116.9 \\
\hline 724.5011 & 17.2 & 92.4 & 167.3 \\
\hline 774.5457 & -79.2 & 91.0 & 193.7 \\
\hline 745.5586 & -169.9 & 87.2 & 239.5 \\
\hline 695.4670 & -149.4 & 84.1 & 237.6 \\
\hline 790.5405 & -409.7 & 82.7 & 768.6 \\
\hline 869.5550 & -166.6 & 82.0 & 66.4 \\
\hline 748.5012 & -153.9 & 80.5 & 214.8 \\
\hline 672.4697 & -116.9 & 78.0 & 35.8 \\
\hline 768.5564 & -308.5 & 76.3 & 336.4 \\
\hline 778.5756 & -85.7 & 75.8 & 56.1 \\
\hline 721.4815 & -89.2 & 74.6 & 197.9 \\
\hline 773.5330 & -92.2 & 74.4 & 44.6 \\
\hline 690.5085 & -167.7 & 68.4 & 75.6 \\
\hline 770.5707 & -158.0 & 67.7 & 274.5 \\
\hline 552.2735 & -209.0 & 63.4 & 202.6 \\
\hline
\end{tabular}




\begin{tabular}{|r|r|r|r|}
\hline 725.5132 & -242.9 & 63.2 & 264.1 \\
\hline 763.5123 & -181.3 & 63.0 & 167.6 \\
\hline 750.5453 & 59.5 & 62.3 & 202.2 \\
\hline 836.5360 & -115.2 & 61.1 & 65.6 \\
\hline 719.4664 & -66.0 & 57.5 & 39.5 \\
\hline 722.5143 & 22.7 & 56.8 & 229.4 \\
\hline 698.4847 & -69.2 & 53.7 & 33.6 \\
\hline 888.5582 & -88.3 & 52.1 & 178.0 \\
\hline 739.5118 & -114.4 & 51.7 & 80.5 \\
\hline 831.5657 & -83.3 & 44.0 & 57.5 \\
\hline 581.3104 & -67.1 & 40.4 & 144.7 \\
\hline 812.5460 & -192.3 & 37.1 & 157.7 \\
\hline 775.5486 & -29.7 & 33.5 & 83.0 \\
\hline 696.4697 & -54.0 & 32.7 & 78.8 \\
\hline 751.5476 & 32.9 & 31.6 & 103.8 \\
\hline 791.5426 & -230.0 & 30.1 & 429.5 \\
\hline 661.4815 & -54.7 & 28.2 & 36.6 \\
\hline 769.5586 & -119.7 & 25.5 & 133.4 \\
\hline 600.3237 & -65.1 & 24.6 & 88.8 \\
\hline 723.5166 & 12.9 & 20.9 & 99.7 \\
\hline 722.4852 & -20.0 & 16.3 & 58.3 \\
\hline 771.5744 & -46.8 & 15.9 & 91.1 \\
\hline 675.4969 & -45.8 & 12.4 & 69.7 \\
\hline 726.5162 & -76.7 & 10.2 & 90.8 \\
\hline 528.2739 & -97.8 & 7.1 & 208.8 \\
\hline 792.5564 & -210.4 & 3.5 & 459.6 \\
\hline 767.5435 & -411.3 & 3.3 & 937.1 \\
\hline 610.2734 & -40.4 & -1.9 & 34.3 \\
\hline 609.2650 & -63.0 & -3.6 & 68.7 \\
\hline 749.5132 & -116.9 & -4.2 & 312.1 \\
\hline 766.5296 & -166.7 & -6.6 & 410.9 \\
\hline 836.5461 & -81.4 & -7.4 & 161.7 \\
\hline 750.5162 & -32.8 & -11.3 & 113.1 \\
\hline 606.2417 & -22.6 & -11.7 & 27.9 \\
\hline 766.5405 & -884.1 & -13.0 & 2071.8 \\
\hline 793.5585 & -94.5 & -14.1 & 228.6 \\
\hline 798.6572 & -49.2 & -16.8 & 80.6 \\
\hline 726.5816 & -52.5 & -22.2 & 97.8 \\
\hline 688.5479 & -19.1 & -27.0 & 129.9 \\
\hline 797.6553 & -124.4 & -29.4 & 209.2 \\
\hline 608.2577 & -52.3 & -30.7 & 70.6 \\
\hline & & & \\
\hline
\end{tabular}




\begin{tabular}{|r|r|r|r|}
616.4718 & -32.2 & -52.4 & 233.7 \\
\hline 687.5455 & -80.7 & -57.5 & 405.3 \\
\hline 838.5615 & -56.5 & -58.1 & 193.1 \\
\hline 795.5742 & -56.7 & -68.5 & 338.3 \\
\hline 794.5709 & -133.2 & -78.2 & 638.4 \\
\hline 751.5290 & -69.1 & -78.6 & 265.7 \\
\hline 702.5164 & -48.7 & -86.8 & 261.2 \\
\hline 789.5486 & -66.9 & -111.7 & 330.5 \\
\hline 701.5135 & -145.9 & -151.8 & 611.8 \\
\hline 788.5450 & -182.4 & -202.6 & 733.6 \\
\hline
\end{tabular}




\section{Supplemental Table 4. Software and data analysis tools for performing this method. From}

left to right the software tool, purpose and location for download or purchase are listed.

\begin{tabular}{|c|c|c|}
\hline Software & Purpose & Location \\
\hline FIJI & $\begin{array}{l}\text { Figure formation, } \\
\text { Image Registration }\end{array}$ & https://fiji.sc/ \\
\hline FlexImaging & $\begin{array}{l}\text { Image Visualization, } \\
\text { Spotlist Generation }\end{array}$ & $\begin{array}{l}\text { https://www.bruker.com/products/mass-spectrometry- } \\
\text { and-separations/ms- } \\
\text { software/fleximaging/overview.html }\end{array}$ \\
\hline QuPath & $\begin{array}{l}\text { Multimodal Image } \\
\text { Data Extraction }\end{array}$ & https://qupath.github.io/ \\
\hline Rstudio & $\begin{array}{l}\text { Segmentation/IMS } \\
\text { Data Analysis, } \\
\text { imzml } \\
\text { Reconfiguring, Data } \\
\text { Extraction }\end{array}$ & https://cran.r-project.org/mirrors.html \\
\hline SCiLS & Exportation to imzml & $\begin{array}{l}\text { https://www.bruker.com/products/mass-spectrometry- } \\
\text { and-separations/ms-software/scils/overview.html }\end{array}$ \\
\hline Image Fusion & Data Mining & https://fusion.vueinnovations.com/ \\
\hline $\begin{array}{l}\text { MSRC } \\
\text { Registration } \\
\text { Toolbox }\end{array}$ & Image Registration & https://github.com/nhpatterson/regtoolboxmsrc \\
\hline Prism & Statistical Analysis & https://www.graphpad.com/scientific-software/prism/ \\
\hline Xcalibur & $\begin{array}{l}\text { LC-MS/MS Data } \\
\text { Analysis }\end{array}$ & $\begin{array}{l}\text { https://www.thermofisher.com/order/catalog/product/ } \\
\text { OPTON-30487 }\end{array}$ \\
\hline LIPIDMAPS & $\begin{array}{l}\text { Lipid Accurate Mass } \\
\text { and MS/MS } \\
\text { Prediction }\end{array}$ & http://www.lipidmaps.org/tools/ms/ \\
\hline
\end{tabular}

